University of Rhode Island

DigitalCommons@URI

Open Access Master's Theses

2020

\title{
A COMPARISON OF OPEN AND CLOSED CHAIN KNEE EXTENSION EXERCISES ON PATTERNS OF QUADRICEPS HYPERTROPHY
}

Domenic Angelino

University of Rhode Island, domenicangelino@gmail.com

Follow this and additional works at: https://digitalcommons.uri.edu/theses

\section{Recommended Citation}

Angelino, Domenic, "A COMPARISON OF OPEN AND CLOSED CHAIN KNEE EXTENSION EXERCISES ON

PATTERNS OF QUADRICEPS HYPERTROPHY" (2020). Open Access Master's Theses. Paper 1845.

https://digitalcommons.uri.edu/theses/1845

This Thesis is brought to you for free and open access by DigitalCommons@URI. It has been accepted for inclusion in Open Access Master's Theses by an authorized administrator of DigitalCommons@URI. For more information, please contact digitalcommons-group@uri.edu. 


\section{A COMPARISON OF OPEN AND CLOSED CHAIN KNEE EXTENSION EXERCISES ON PATTERNS OF QUADRICEPS HYPERTROPHY \\ BY \\ DOMENIC ANGELINO}

\section{A THESIS SUBMITTED IN PARTIAL FULFILLMENT OF THE REQUIREMENTS FOR THE DEGREE OF \\ MASTER OF SCIENCE}

IN

KINESIOLOGY 
MASTER OF SCIENCE THESIS

OF

DOMENIC ANGELINO

APPROVED:

Thesis Committee:

$\begin{array}{ll}\text { Major Professor } & \text { Jacob Earp } \\ & \text { Disa Hatfield }\end{array}$

Kathleen Melanson

Nasser H. Zawia

DEAN OF THE GRADUATE SCHOOL

UNIVERSITY OF RHODE ISLAND

2020 


\section{ABSTRACT}

Resistance training causes hypertrophy, however, the magnitude of muscle growth varies along the length of the muscle (i.e. proximo-distally). For running based athletes and those dependent on movement about the hip, preferential proximal hypertrophy of the quadriceps femoris (the primary knee extensor) shifts the center of mass (CoM) of the thigh closer to the hip which provides a direct biomechanical advantage by decreasing the moment of inertia of the high about the hip (I). This in turn can increase movement velocity and economy and has been observed in studies using mathematical modeling and when comparing elite national level sprinters. Recent studies have reported that the pattern of quadriceps hypertrophy differs between different types of training (plyometrics vs traditional heavy resistance training) or when different types of contractions (eccentric vs concentric) are performed. However, no study to date has explored how exercise selection affects patterns of hypertrophy. Therefore, the purpose of the present study was to compare the effects of open kinetic chain (OKC) and closed kinetic chain (CKC) exercises on quadriceps patterns of hypertrophy and to determine if patterns of hypertrophy differ and if so does this result in a significant effect on CoM and I. Given pilot data from our lab, we hypothesized that CKC would result in similar proximal hypertrophy but less distal hypertrophy of the quadriceps compared to OKC, thus shifting CoM proximally and decreasing I about the hip. To test our hypothesis, 12 untrained participants (male =7; female $=5)$ aged $18-35$ years participated in an 8 week resistance training 
intervention where each participant trained by performing both unilateral CKC (squat) and OKC (knee extension) exercises on separate legs. Before and after the training program MRI of the quadriceps femoris was performed in order to measure changes in muscle cross sectional area in the proximal-thigh (1/3 thigh length), mid-thigh (1/2 thigh length) and distal-thigh (2/3 thigh length). Regional cross sectional area of the quadriceps femoris was compared between exercises and over time using a $2 \times 2$ mixed model ANOVA with Bonferoni post-hoc corrections. Results revealed that both conditions resulted in an increase in muscle volume which was similar between conditions $\left(C K C \Delta 60.2 \pm 110.5 \mathrm{~cm}^{3}\right.$, OKC $\Delta 79.5 \pm 87.9 \mathrm{~cm}^{3}, \mathrm{p}=$ 0.285). However, the pattern of hypertrophy differed along the length of the thigh and between conditions with CKC experiencing a significant increase in cross sectional area in only the distal-thigh region $(p=0.044)$ and OKC experiencing a significant increase in both the mid- and distal-thigh regions ( $p$ $=0.003-0.004)$. Additionally, a significant interaction effect of exercise and time was observed for CoM $(p<0.001)$ and $I(p<0.001)$, where CKC resulted in CoM shifting proximally and / reducing about the hip when compared to OKC. Given running and other athletes can benefit from a proximal shift in CoM of the thigh and reduced I of the thigh about the hip, our results suggest that running based athletes should preferentially select CKC exercises over OKC exercises during their resistance training program. 


\section{ACKNOWLEDGMENTS}

I am very appreciative of the opportunity provided to me to pursue my graduate education and complete this thesis. I am grateful to both Dr. Delmonico and Dr. Earp whom had accepted me into the M.S. Kinesiology program, and am grateful for their help and guidance along the way in my studies. Dr. Earp has helped me do many things I never thought I would do and given me many opportunities to learn and excel, which have helped me grow into being capable enough to produce my thesis. Dr. Earp has helped me with development of research skills, teaching skills, has taught me much academically, and has helped provide valuable guidance in life.

I would also like to thank Robert Lavelle for his assistance in administering the intervention. A further thank you goes out to all the undergraduate Kinesiology students who had helped with research and intervention administration at any and all stages in the completion of this project.

I additionally want to thank Christine Huynh for her all-consuming and warm support. Finally, l'd especially like to thank my friends Darrel Ros, Veenlon Peang, and Keelin Howard for supporting me throughout graduate school. 


\section{TABLE OF CONTENTS}

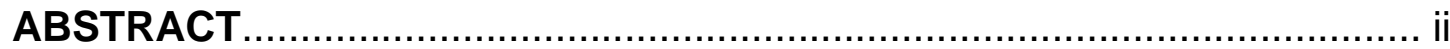

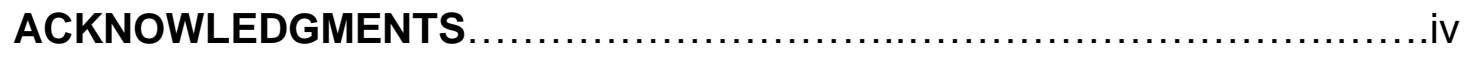

TABLE OF CONTENTS $\ldots \ldots \ldots \ldots \ldots \ldots \ldots \ldots \ldots \ldots \ldots \ldots \ldots \ldots \ldots \ldots \ldots \ldots \ldots \ldots \ldots \ldots$

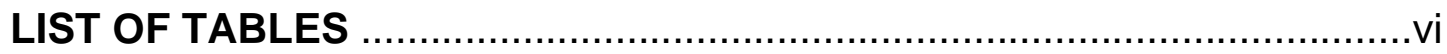

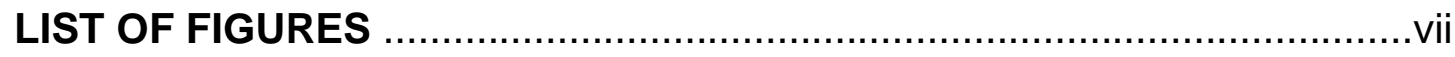

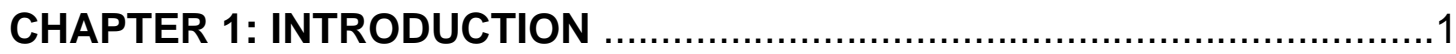

CHAPTER 2: REVIEW OF LITERATURE $\ldots \ldots \ldots \ldots \ldots \ldots \ldots \ldots \ldots \ldots \ldots \ldots \ldots \ldots 14$

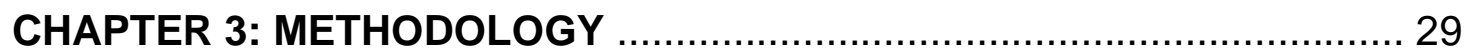

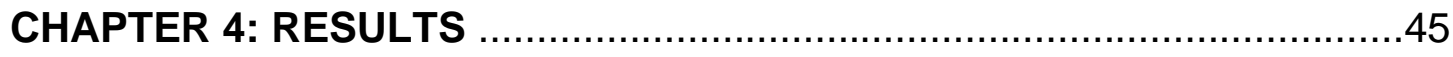

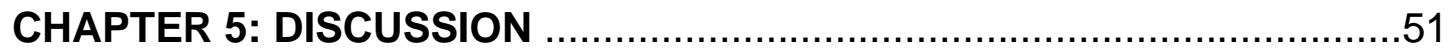

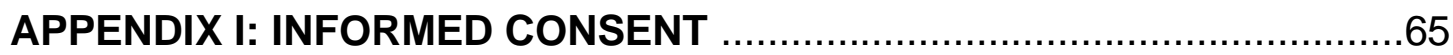

APPENDIX II: HEALTH HISTORY QUESTIONAIRRE .......................76

APPENDIX III: TWO DAY FOOD RECORD $\ldots \ldots \ldots \ldots \ldots \ldots \ldots \ldots \ldots \ldots \ldots \ldots \ldots . \ldots \ldots$

APPENDIX IV: DATA COLLECTION SHEET $\ldots \ldots \ldots \ldots \ldots \ldots \ldots \ldots \ldots \ldots \ldots \ldots . \ldots 1$

APPENDIX V: INTERVENTION AND STRENGTH TESTING DATA

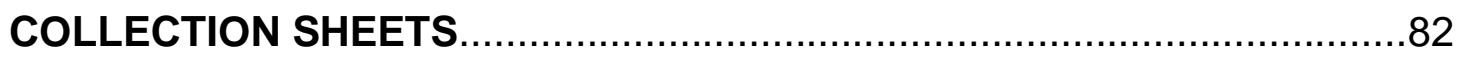

APPENDIX VI: BORG CR-10 SCALE OF PERCEIVED EXERTION ..........103

APPENDIX VII: EMAIL RECRUITMENT SCRIPT.............................105

APPENDIX VIII: RECRUITMENT FLYER $\ldots \ldots \ldots \ldots \ldots \ldots \ldots \ldots \ldots \ldots \ldots \ldots \ldots \ldots 106$

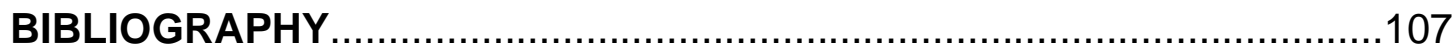




\section{LIST OF TABLES}

TABLE

PAGE

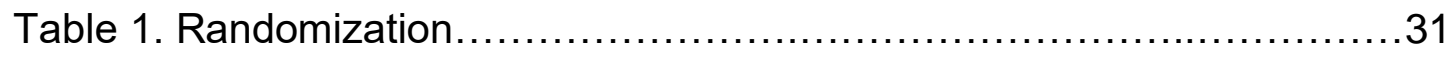

Table 2. RM testing protocol.................................................

Table 3. 8 week intervention periodization..................................42

Table 4. NSCA guidelines for resistance training status.....................42

Table 5. NSCA guidelines for resistance training frequency ...................43

Table 6. NSCA guidelines for resistance training targeted workout

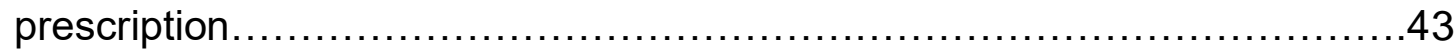

Table 7. Cohort characteristics ............................................... 


\section{LIST OF FIGURES}

FIGURE

PAGE

Figure 1. The influence of thigh CoM on / about the hip....................... 4

Figure 2. Resistance during early-swing phase of running gait................4

Figure 3. Resistance during mid-swing phase of running gait..................5

Figure 4. Differences between proximal (left) and distal (right) regional activation of hamstring muscles between OKC and CKC

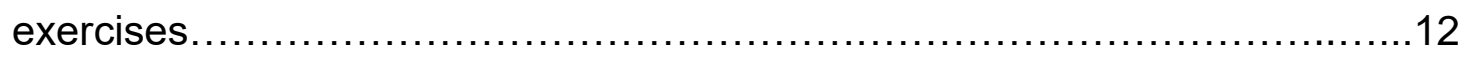

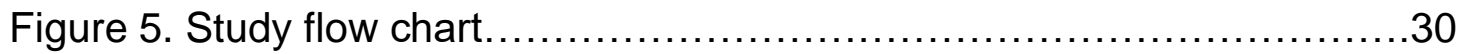

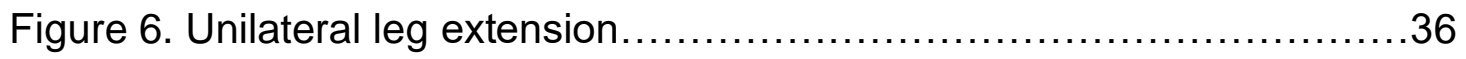

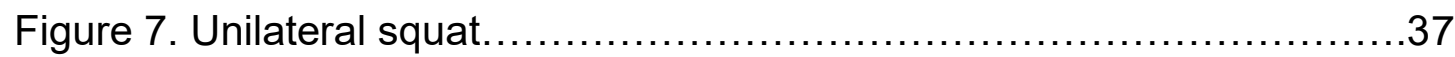

Figure 8. Absolute strength (left) and relative strength (right) $\ldots \ldots \ldots \ldots \ldots \ldots . \ldots 4$

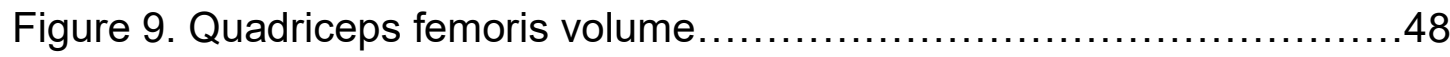

Figure 10. Quadriceps femoris CSA following CKC (left) and OKC (right)

training at the proximal, middle, and distal

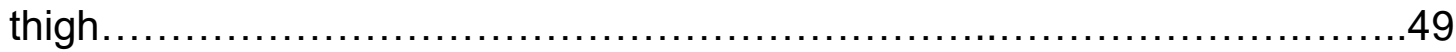

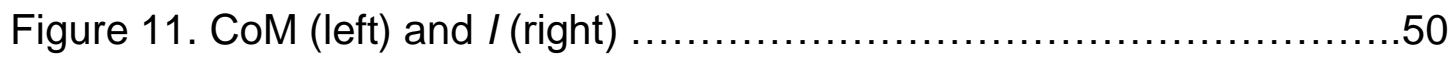




\section{CHAPTER 1}

\section{INTRODUCTION}

Resistance training that involves active knee extension results in hypertrophy of the quadriceps femoris muscle group (1-4). This hypertrophy can be beneficial to athletic performance given that greater cross sectional area of muscle yields greater force production potential during many common movements in sport such as sprinting, jumping and changing direction (2, 48). However, hypertrophy also negatively affects such movements, as hypertrophy results in an increase in mass, which is the primary form of resistance that must be overcome in these movements in the form of inertia (linear motion) and moment of inertia (for angular motion: 9). Therefore, the positive benefits of the added force production are balanced between the negative effects of the added mass. In response to this, a growing area of research has focused on means by which athletes can increase force production potential of muscles while minimizing the negative effects of mass related to hypertrophy. Selective or targeted regional hypertrophy is one such solution.

When considering the quadriceps femoris, the largest muscle group in the body by mass, hypertrophy provides a direct benefit to force production at the knee but this added mass increases the resistance that needs to be overcome 
for hip motion to occur. This resistance is referred to as the moment of inertia of the thigh about the hip (or I). Movement around the hip joint is important for athletic performance given many sports rely on hip dominant movements such as running $(8,10)$. As the moment of inertia $(I)$ of the hip is the product of the mass of the hip multiplied by the location of the center of mass squared of the hip $\left(I=m r^{2}\right)$ the location at which hypertrophy occurs is exponentially more important mathematically than simply how much mass is increased. Therefore, hypertrophy that is more proximal to the hip will minimize the resistance the athlete needs to overcome while running thereby increasing how fast the limb can be moved (angular acceleration = torque / moment of inertia) decreasing energy costs more so than when the same amount of hypertrophy occurs closer to the knee / distally $(1,2,6,10)$.

As distribution of hypertrophy along a muscle may have important implications for athletic performance, creating resistance training practices that provide this direct biomechanical benefit may be of great importance for running athletes. Recent research has suggested that it is possible to manipulate changes in the location of mass along the length of a muscle via manipulation of various acute programming variables in exercise prescription $(2,11-14)$. However, it is presently unknown how exercise selection affects patterns of hypertrophy. Specifically, it is of interest to determine if training with open chained exercises (in which the foot moves freely around the knee, e.g. a knee extension) compared to closed chain exercises (where the feet are fixed and 
the body is moved, e.g. a squat) results in meaningful changes in muscle mass distribution, center of mass (CoM), and moment of inertia ( $I$ ) about the hip as these are variables within a training program that can be easily manipulated and controlled.

Given CoM is a function of morphological conditions within muscle (i.e. the shape of the muscle), the overall distribution of mass will alter the location of CoM $(2,10,15,16)$. Changes in the location of CoM will subsequently alter I about the hip $\left(I=m r^{2}\right.$, where $I=I$ about the origin, $m=$ mass, and $r^{2}=$ radius of CoM squared) (See figure 1). This is important as a smaller / increases the speed at which the hip can be moved $\left(\alpha=\mathrm{T}^{-1}\right.$, where $\alpha=$ angular acceleration, $\mathrm{T}=$ the maximum torque that can be produced by the hip muscles and $I=$ moment of inertia). Therefore by reducing $I$ the hip can attain higher angular velocities during a variety of motions such as running, thus improving athletic performance (6-8). This is most evident when considering rotation about the hip joint given its role in common athletic movements such as general locomotion, sprinting, jumping, and kicking (8). For example, figures $2 \& 3$ depict the effect of shifting CoM of the thigh proximally by $2 \mathrm{~cm}$ on I at the two extreme positions of the leg during swing phase of running for an average US male using Dempster anatomical models to determine predicted anthropometrics. These figures illustrate that the $2 \mathrm{~cm}$ shift in CoM would reduce / between $2.0 \%$ and $4.9 \%$ throughout the stance phase of running. 
Figure 1. The Influence of Thigh CoM on I About the Hip

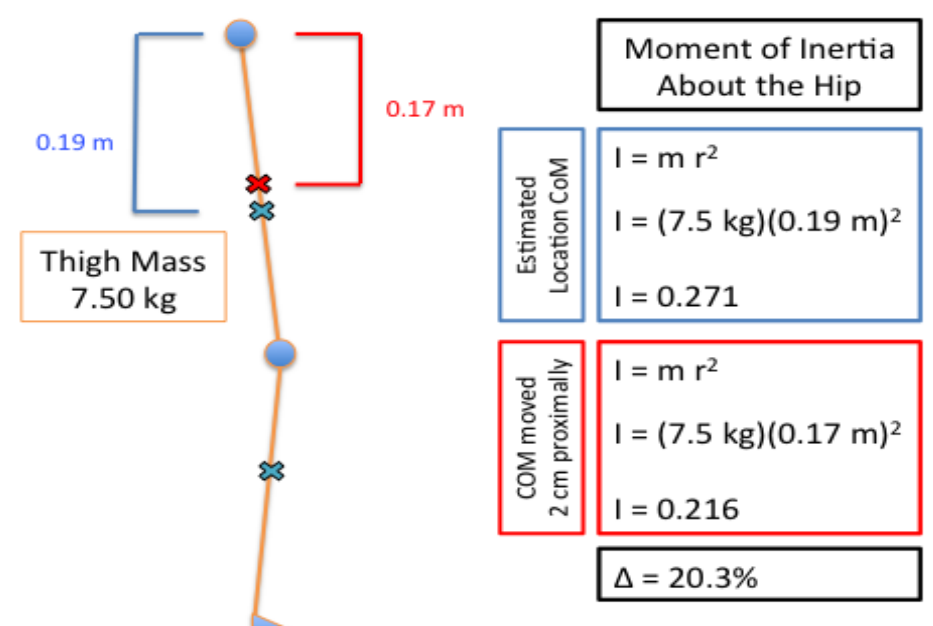

Figure 2. Resistance During Early-Swing Phase of Running Gait

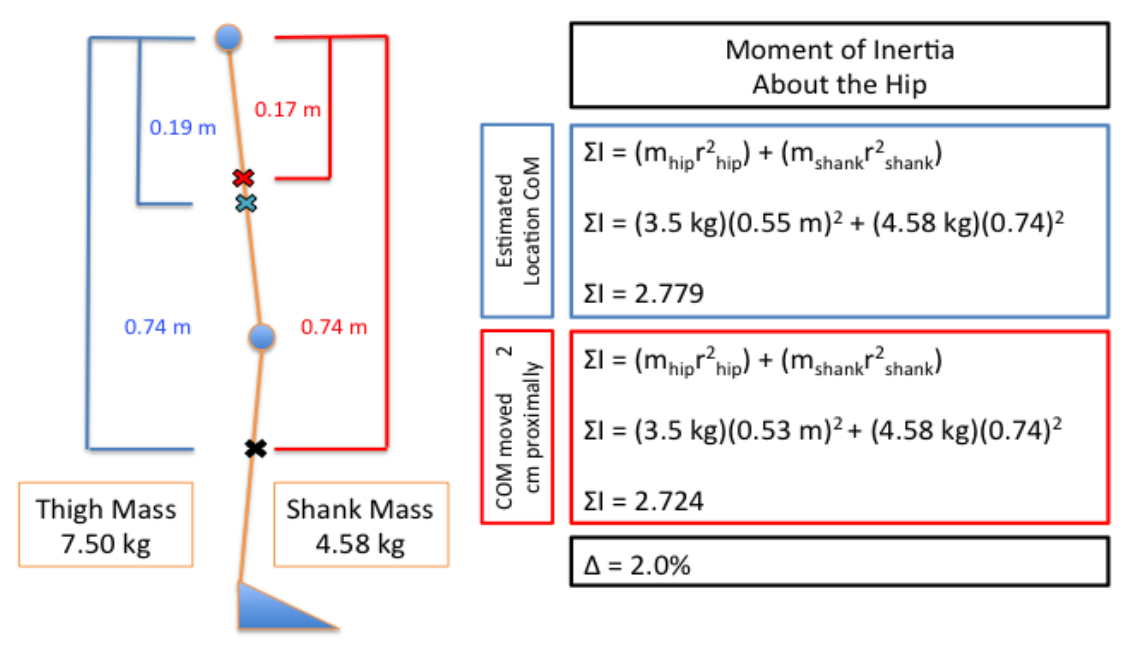


Figure 3. Resistance During Mid-Swing Phase of Running Gait
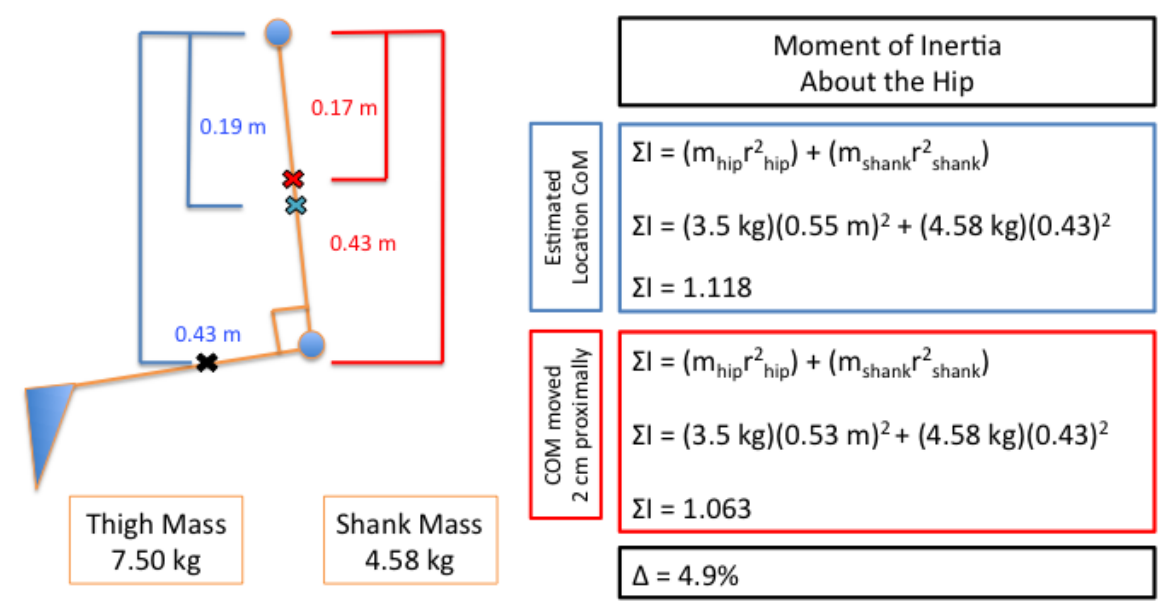

Figures 1,2,3. The above figures model the effects of a $2 \mathrm{~cm}$ proximal shift in CoM of the thigh shank on I about the hip during various phases of running gait. Original position is depicted in blue and post-adaptation position is depicted in red. Given the exponential relationship between CoM and I, minor changes in CoM result in more profound changes in $I$.

A smaller I also reduces the amount of torque necessary to move at a given velocity $(T=/ \alpha$, where $T$ is the torque necessary to complete the movement, I $=$ moment of inertia, and $\alpha=$ the acceleration necessary to complete a task) $(8,10,16)$. The equation for $I$ the location of CoM is squared unlike the mass therefore / encountered is more greatly influenced by location of changes in mass than magnitude of the change in mass $(15,16)$. Thereby movement efficiency can also be increased by proximally shifting CoM given less muscle force will be required to generate movement about the hip with a reduced thigh 
resistance moment, potentially limiting and mitigating accumulated muscular fatigue during performance.

Being able to run faster and more efficiently can improve performance in a meaningful way for diverse groups of athletes. Thus, training that results in a more proximal shift in CoM of the thigh would be most preferable for those populations. Given CoM of the thigh is primarily influenced by the mass and shape of the quadriceps femoris muscle, and that muscle can undergo significant and inhomogeneous hypertrophy, controlling that pattern can result in directly improving athletic performance. $(2,10,11,15,16)$.

Until recently, skeletal muscle hypertrophy had been assumed to be homogenous, with hypertrophy distribution occurring proportional to the muscle thickness of a region in response to muscle-growth inducing stimuli ( 3 , 12, 17). This assumption thus implied relative hypertrophy was consistent, though absolute hypertrophy differed by muscle region. Recent published works have contested this, suggesting that muscles may experience hypertrophy in an inhomogeneous fashion along the length of a muscle (2, 11-14). For instance, it has been demonstrated that hypertrophy occurs in an inhomogeneous manner following 12 week of isokinetic knee extension training, with mass distribution varying between the proximal and distal regions of the quadriceps femoris (2). This notion is further supported by the work of Wakahara et al. (17), who reported that hypertrophy occurs in an 
inhomogeneous fashion along the length of the triceps brachii, which experiences greater proximal hypertrophy from closed chain exercise. However, as the proximal portion of the triceps brachii (the long head) has a role at both the shoulder and elbow, the authors assumed this selective activation of this part of the muscle due to its role at the shoulder was the driving mechanism for their results and therefore their results may apply to the biarticular rectus femoris muscle of the quadriceps femoris.

Inhomogeneity may also be influenced by manipulation of acute programming variables for exercise prescription. A study by Earp et. al (11) found that manipulating training load and movement velocities in the back squat exercise changed the pattern of hypertrophy of the quadriceps femoris. The authors reported that slow-speed heavy-load training resulted in greater proximal hypertrophy and moderate-load high-velocity training resulted in more distal hypertrophy. This suggests that muscle mass distribution induced by a hypertrophy-causing stimulus may vary dependent on varied prescription parameters. Given patterns of hypertrophy may be influenced by training, it may be the case that altering other training parameters can alter these patterns as well. The alterable patters of inhomogeneity of hypertrophy within the quadriceps femoris may be influenced via both OKC (knee extension) and CKC (back squat) exercises. 
Differences between OKC and CKC exercises on hypertrophy localization can be reasonably expected $(1,3,18)$. Previous research evaluating electrical activity of the quadriceps femoris during various knee extensor and hip flexor exercises suggests that the rectus femoris muscle may experience hypertrophy in an inhomogeneous manner in response to a single exercise due to its biarticular nature (13). The rectus femoris portion of the muscle is located more proximally than the vastus lateralis, vastus intermedius, and vastus medialis aspects of the muscle (3). Rectus femoris activation has been suggested to be less than that of the other quadriceps muscles when the hip is flexed, resulting in greater distal quadriceps femoris muscle activation and similar activation in the proximal and middle sections of the quadriceps femoris $(4,10,13)$. The degree of hip flexion at different points in the range of motion during an exercise involving knee extension joint action may alter involvement of the rectus femoris and as such result in varied hypertrophy of the proximal quadriceps femoris. This may therefore result in differing hypertrophy localization along the length of the quadriceps femoris muscle.

Given OKC exercises involving the quadriceps femoris such as the knee extension involve the hip being positioned in a fixed location and CKC exercises involving the hip moving through a full range of motion, it is reasonable to expect there to be observable differences in induced hypertrophy between interventions involving one of the two types of exercises. Importantly, the degree of hip flexion at the point where resistance torque is 
greatest for each exercise differs and as such the rectus femoris would receive differing stimulus for growth (18-20). However, further changes may be driven by inter and intramuscular architectural differences that elicit different training responses under the conditions of different imposed demands. The actual manifestation of changes however is currently unknown given no past study had explored the differences between $\mathrm{OKC}$ and $\mathrm{CKC}$ exercise training on quadriceps femoris muscle morphology.

Conversely, past research has supported that the most distal fibers of the vastus medialis, the vastus medialis oblique (commonly called the VMO) cannot be preferentially activated by altering hip joint position, and thus the vastus lateralis experiences similar hypertrophy to the vastus medialis oblique independent of orientation of the hip (21). This suggests that differences in hypertrophy localization between the vastus lateralis and vastus medialis oblique may be attributed to internal factors rather than hip position. It has been suggested that differences in muscle architecture exist between the vastus lateralis, vastus medialis, rectus femoris, and vastus intermedius (3). These differences manifest in muscle thickness along the muscle's length, fascicle length, and fascicle angle, which may result in differing muscle activation and thereby hypertrophy localization differences between open and closed chain exercises $(3,12,22,23)$. Furthermore, recent work has supported that the recruitment of mechanically favorable motor units, which best address task specific demands, may differ within and between regions of 
a muscle dependent upon the task being executed (24). The recruited task specific motor units may circumnavigate the size principle of motor neuron recruitment, allowing for higher order neurons to be recruited at lower intensities than their typical force threshold during certain tasks. This suggests that motor unit recruitment and thereby location of hypertrophy in the quadriceps femoris may differ between $\mathrm{OKC}$ and $\mathrm{CKC}$ exercise tasks given they are mechanically different. This suggested mechanism for adaptation may drive inhomogeneity and provide a basis for controlling patterns of it. The actual manifestation of changes however is currently unknown given no past study had explored the differences between OKC and CKC exercise training on quadriceps femoris muscle morphology.

Manipulating patterns of hypertrophy may also benefit physical therapy and rehabilitation practices in addition to athletic performance. Conditions such as patellofemoral syndrome have been shown to lead to localized atrophy in either the proximal or distal aspect of the quadriceps femoris muscle group (25). Manipulation of programming variables to more effectively target hypertrophy localization may further support these practices. It has been demonstrated that CKC and OKC exercises are not equally effective in treating patellofemoral pain syndrome $(1,26)$. A basis for the differing benefit of different types of exercises on treating patellofemoral syndrome has already been developed, though developing an understanding of the effects of OKC 
and CKC exercise intervention may help target exercise prescription treatments for other quadriceps femoris atrophy related issues.

Pilot data for the current study supports that closed chain exercise involves greater proximal regional activation of thigh musculature and similar distal activation when contrasted with open chain exercise. Unpublished pilot data from our lab collected by Andrew Sherman M.S., compared regional activation of the hamstring muscle group during open and closed chain exercises using near-infrared spectroscopy. In this study it was observed that open chain lying leg curls resulted in significantly more activation in the distal portion of the hamstring muscle than the closed kinetic chain Nordic hamstring exercise (See figure 4). Though the involved muscle group differs from the quadriceps femoris, this supports that imposing different task specific demands in the form of OKC and CKC exercise may elicit different hypertrophy responses in skeletal muscle. Differences in regional muscle activation have been supported to shift hypertrophy localization during a training intervention to the region with the greatest activity $(2,12,13)$. This is in line with the findings of Wakahara et al. (17), whose research supported closed chain exercise inducing greater proximal hypertrophy in the triceps brachii, but whose results were attributed to activation of the long head of that muscle, which is known to have separate activation pathway to the rest of the triceps brachii muscle. 


\section{Regional Exercise Demands}

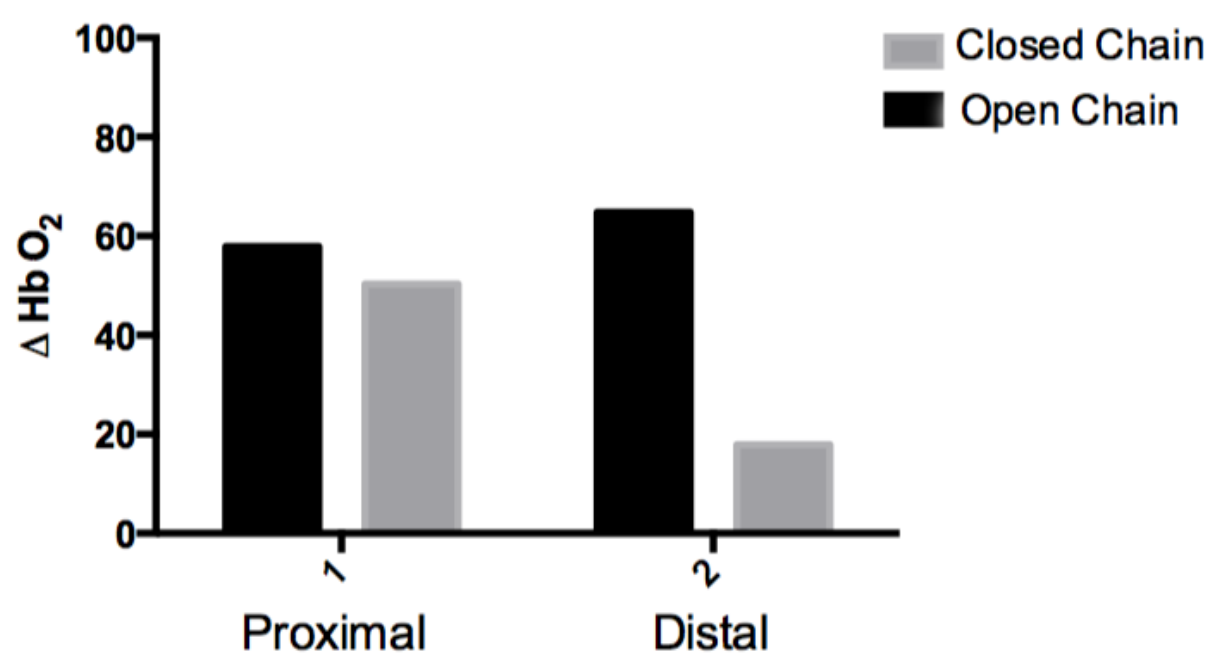

Figure 4. Pilot data collected by Andrew Sherman M.S. suggesting differences between proximal (left) and distal (right) regional activation of hamstring muscles between OKC and CKC exercises as measured via near-infrared spectroscopy, as represented by change in oxygen saturation \% of Hemoglobin $\left(\mathrm{Hb} \mathrm{O}_{2}\right)$. Differences in activation were recorded acutely and were not reflective of an observed training adaptation in the pilot data.

The current study is the first to directly explore the effects of OKC and CKC extension exercises on quadriceps femoris muscle morphology via a training intervention. Developing a successful training model for influencing inhomogeneous hypertrophy and muscle mass distribution within the quadriceps will allow for more targeted and effective exercise prescription in both the athletic and rehabilitative sectors. Development of an intervention training model allows for both an exploration of mechanisms and effects as well as a template for evidence-based professionals to utilize in their own 
practices. Therefore, the purpose of the current study was to determine if regional hypertrophy of the quadriceps femoris and mechanical parameters differ between OKC and CKC knee extensor exercises. We hypothesized that CKC knee extensor training will result in similar proximal quadriceps femoris hypertrophy but less hypertrophy of the distal quadriceps femoris when compared to OKC knee extensor training, thus shifting CoM more proximally and decreasing / about the hip. 


\section{CHAPTER 2}

\section{REVIEW OF LITERATURE}

\section{Inhomogeneous Hypertrophy}

A study conducted by Miyamoto et al. (13) explored differences in hypertrophy localization (proximal vs. distal) within the rectus femoris muscle of the quadriceps femoris. Participants were inactive and had not engaged in habitual resistance training within the most recent 6 months. Participants performed unilateral knee extension and hip flexion only tasks at varying intensities while EMG probes were positioned at 30\%, 50\%, 70\%, and 90\% lengths of the thigh over the rectus femoris, vastus lateralis, and vastus medialis muscles, the vastus intermedius was excluded because it was too deep to accurately measure via EMG. Hip flexion tasks were performed in addition to knee extension tasks to evaluate differences in rectus femoris muscle activation and explore passive insufficiency in the biarticular muscle. It was observed that rectus femoris activity ratio of activation (hip flexion task: knee extension task) were $55.5 \pm 17.0 \%$ (distal region), $73.7 \pm 16.1 \%$ (middle region), and $80.1 \pm 17.2 \%$ (proximal region), with activation being greater during knee extension than hip flexion. Rectus femoris activation did not differ significantly between regions during the knee extension trials, however differed significantly during the hip flexion trials, with distal activation being smaller than both proximal and middle regions of the rectus femoris $(P<0.05)$. 
A potential limitation of this study relates to the use of EMG given the measurement tool records signals from motor unit endplates and the distance between the receiver and the end plate may result in a lapse in time between the occurrence of muscle activity and its recording. This delay may have confounded findings given points of peak activation may have been assessed to occur later in the range of motion of a given exercise than they had actually occurred. The findings however, support the hypothesis of the present study.

A separate study conducted by Mitchell et al. (27) explored anatomical differences between proximal and distal portions of the vastus medialis muscle. A dissection of 50 cadavers (age $=61 \pm 21$ years old) was conducted on cadavers with no history or evidence of musculoskeletal pathology, or osteoarthritis. Left thighs were fully dissected and muscle fiber orientation, nerve supply, fascicle planes between fibers were assessed separately in both the proximal and distal portions of the vastus medialis muscle. It was found that the angular orientation of proximal fibers significantly differed from that of distal fibers $\left(11.46 \pm 2.96^{\circ}: 52.20 \pm 6.20^{\circ} ; p<0.001\right)$. The authors concluded that this finding suggests the proximal portion of the vastus medialis has a greater role in performing knee extension, and the distal portion is more involved in stabilizing the patella. These differences in function between proximal and distal portions of the vastus medialis support inhomogeneity of hypertrophy along its length. Thus, these findings are important given they support the existence of inhomogeneity across the length of a muscle. 
A 12 week intervention study conducted by Ema et al. (12) explored the morphological changes to the quadriceps and each of its four regions in response to a seated knee extension resistance training program. The protocol involved a range of motion between $20^{\circ}$ and $110^{\circ}$ of flexion during each knee extension rep and intensity was set to a constant $80 \%$ one repetition maximum for each working set, with one repetition maximum being retested each two weeks for prescription purposes. Ultrasound and MRI imaging were performed at $15 \%, 35 \%, 50 \%, 55 \%$, and $70 \%$ the length of the thigh as a basis for analysis of regional hypertrophy of the quadriceps femoris and its patterns. Hypertrophy occurred in an inhomogeneous manner along the length of the quadriceps femoris with relative change in cross sectional area of the vastus lateralis and rectus femoris differing between proximal and distal regions after the resistance training intervention $(p<0.05)$. It was found that overall greater hypertrophy occurred within the rectus femoris than the vastus lateralis and medialis muscles. However, greater distal hypertrophy occurred than proximal in all regions of the quadriceps femoris via the seated knee extension protocol. This supports the present study's hypothesis.

Another study conducted by the lab of Wakahara \& Ema et al. (14) explored the association between regional muscle activation as assessed by EMG and regional hypertrophy caused by a 12 week knee extension intervention as a follow up to their previous study on inhomogeneity of hypertrophy in the 
quadriceps femoris. MRI scans were used pre and post intervention to assess volume and muscle cross sectional area of the quadriceps femoris and each of its muscles at differing percentages of the total length of the thigh. EMG measurements were taken at each site during working sets of knee extension to assess muscle activation differences. The researchers found that muscle activation has a strong association with training induced hypertrophy localization and quadriceps femoris morphology $(p<0.05)$, suggesting that activated tissue results in hypertrophy. This supports the ability of acute quadriceps femoris activation studies to provide valid insight into hypertrophic responses in longitudinal training studies over a duration of up to 12 weeks, as well as the influence of task specific motor unit recruitment on driving inhomogeneity of hypertrophy.

Morphological changes within the quadriceps femoris induced by a knee extension training program were further supported by a 10 week intervention trial conducted by Hakkinen et al. (2), and supported induced inhomogeneity of hypertrophy in both old (61 \pm 4 years) and young ( $29 \pm 5$ years) populations. Vastus medialis, vastus lateralis, vastus intermedius, and rectus femoris regional cross sectional area of muscle increased significantly for both age groups (average regional cross sectional area increase of quadriceps femoris muscles of $\left.8-40 \mathrm{~cm}^{2} ; \mathrm{p}<0.05\right)$, with no significant differences between age groups for cross sectional area changes in any of the regions of the muscle. This suggests that meaningful hypertrophy was induced in each region of the 
quadriceps femoris, however inhomogeneity of hypertrophic responses to an exercise stimulus was not a function of age, according to this research study. This is important when considering and evaluating participant selection criteria of a study.

A study by Blazevich et al. (3) investigated internal architectural factors within each muscular compartment of the quadriceps femoris and the potential for differential muscle fascicle strain encountered by each region dependent upon its individual architecture. Thirty-one sedentary non-habitually resistance trained participants of both genders were recruited to undergo MRI imaging of each compartment of their quadriceps femoris. Muscle architecture was imaged in vivo to identify muscular thickness at differing lengths of each region, fascicle length, and fascicle angle of each region. The researchers had found that architecture differed between the vastus lateralis, vastus medialis, and rectus femoris $(p<0.05)$. These differences in architecture along with fascicle length and angle suggest that fascicle strain would differ between muscular compartments depending on the resistance training stimulus used in an intervention, suggesting hypertrophy localization may differ between open and closed chain knee extension exercises, supporting the premise of the present study.

A study by Earp et al. (11) has explored differences in proximal and distal hypertrophy within the quadriceps femoris in response to different exercise 
prescriptions. The study included 3 training conditions and a control condition for participants $(n=36)$ who do not habitually resistance train over an 8 week

period. All training conditions involved the CKC back squat movement pattern and participants were assigned to either a parallel depth heavy squat, parallel depth jump squat, volitional depth jump squat or no resistance training program condition. The researchers found that hypertrophy was inhomogeneous as a function of the specific exercise prescription used. Specifically, high velocity parallel jump squatting was the only condition to experience a significant proximal increase in quadriceps femoris muscle cross sectional area $(p<0.05)$, and heavy squatting to parallel depth was the only condition that induced significant hypertrophy at the mid thigh $(P<0.05)$. The authors concluded that heavy squat intervention at a parallel depth increases proximal quadriceps femoris cross sectional area greater than jump squatting to parallel, which experienced greater distal increases in muscle cross sectional area. The current study supports the role of exercise type selection in influencing muscle architecture and morphology of the quadriceps femoris during a resistance training intervention. This is important to the present study given differences are hypothesized to be observed by manipulation of exercise type, an acute exercise programming variable.

\section{OKC and CKC Exercises and Movement Mechanics}


An investigation by Wilk et al. (28) compared tibiofemoral joint forces and muscle activity between OKC and CKC movements about the hip and knee joint. This investigation aimed to determine functional differences between the two categories of movements in application to the muscles of the thigh, inclusive of the quadriceps femoris, and associated joints. The study had involved 3 exercise conditions performed with a load equivalent to each participant's $(n=10 ; 11$ or more years of regular resistance training experience) 12 repetition maximum. Participants performed either the seated leg extension, back squat, or leg press exercise with a full range of motion. EMG electrodes were placed on the participants' quadriceps femoris and hamstring muscles, while external loads were measured by an external force plate and motion analysis software developed three dimensional video recordings for data analysis. It was found that maximal compressive force (Squat: $6139 \pm 1709 \mathrm{~N}$ at $91 \pm 15^{\circ}$ Knee flexion; Knee extension: $4598 \pm$ $2547 \mathrm{~N}$ at $75 \pm 13^{\circ}$ Knee flexion, $\mathrm{P}<0.05$ ), maximal posterior shear force (Squat: $1783 \pm 634 \mathrm{~N}$ at $90 \pm 17^{\circ}$ Knee flexion; Knee extension: $1178 \pm 594 \mathrm{~N}$ at $91 \pm 9^{\circ}$ Knee flexion, $P<0.05$ ), maximum anterior shear force (Squat: 0.00 $\mathrm{N}$; Knee extension: $248 \pm 259 \mathrm{~N}$ at $14 \pm 2^{\circ}$ Knee flexion, $\left.\mathrm{P}<0.05\right)$, and maximal external torque (Squat: $150 \pm 40 \mathrm{~N}$ at $78 \pm 12^{\circ}$ Knee flexion; Knee extension: $200 \pm 120 \mathrm{~N}$ at $63 \pm 12^{\circ}$ Knee flexion, $\mathrm{P}<0.05$ ) occur at significantly different angles and with significantly different $\mathrm{N}$ and $\mathrm{Nm}$ resistance forces encountered at those angles while training at the same intensity. Quadriceps femoris activity as a percentage of maximum voluntary 
contraction significantly differed between the squat and the knee extension exercises in the vastus medialis (Squat: $61 \pm 12 \%$; Knee extension: $46 \pm 14 \%$, $P<0.05)$ but not the rectus femoris or vastus lateralis, the vastus intermedius was not assessed because it is a deep muscle. This study conveys that OKC and $\mathrm{CKC}$ exercises involving movement about the knee joint result in the encountering of differed joint forces at different portions of the range of motion, with magnitude of forces varying. This difference in the location of this encountered torque will also influence angular momentum and movement velocity. Differing movement velocities induce muscle fascicle strain differentially dependent on fascicle angle and length, which differ in each muscular compartment and region of the quadriceps femoris (3). Resultantly, quadriceps femoris muscle activation may differ by execution of CKC vs. OKC exercise.

A study by Stensdotter et al. (18) further supported differences in muscle activation of the quadriceps femoris during execution of OKC vs. CKC lower body exercises. The study explored differences in the activation of each of the four quadriceps femoris regions and the time at which each muscle activates during OKC and CKC exercises. Healthy untrained participants $(\mathrm{n}=10$ males and females; age $=28.5 \pm 0.7$ years) performed various $\mathrm{OKC}$ and $\mathrm{CKC}$ knee extension tasks with electromyography probes recording muscle activation. The study found that in CKC knee extension joint action there were no significant differences in time of activation for the vastus medialis, vastus 
intermedius, vastus lateralis, or rectus femoris. This suggests that the muscles act simultaneously in $\mathrm{CKC}$ conditions. In OKC conditions the rectus femoris activates initially, followed by the vastus lateralis and vastus intermedius simultaneously and the vastus medialis activating later $(7 \pm 13 \mathrm{~ms}$ after rectus femoris activation, $\mathrm{P}<0.05)$. Muscle activation amplitude as a percent of maximal voluntary contraction was found to be significantly different for the vastus medialis between the closed chain ( $46 \pm 4.3 \%$ vs. $40 \pm 3.0 \%, P<0.05$ ). This work further suggests that activation patterns of regions of the quadriceps femoris muscle differ with differing movement type conditions, thus suggesting hypertrophy localization may differ from a CKC vs OKC training program.

A novel investigation by Azizi (29) into the occurrence of variable muscle gearing explored changes in muscle fascicle length and angle due to different training stimuli. Participants performed contractions of varying velocities and forces, resulting in changes in fascicle length, angle, and muscle thickness in each condition. It was found that greater fiber rotation occurs in low force high velocity movements, and lesser fiber rotation occurs in high force low velocity movements $(P<0.05)$. This suggests that the internal environment of a muscular compartment is dynamic and may be influenced by the type of stimuli encountered. If an $\mathrm{OKC}$ or $\mathrm{CKC}$ exercise results in increasing the amount of muscle force produced within muscles of the quadriceps femoris differentially, then they will experience different muscle fascicle orientations and architecture intraset. Thus, they will be differentially susceptible to muscle 
fascicle strain, a known driver of hypertrophy. This may result in driving inhomogeneous hypertrophy across the length of the quadriceps femoris.

An investigation by Browning et al. (15) had explored the biomechanical and energetic effects of increasing thigh and leg mass. The investigation aimed to understand the differences between net metabolic rate, movement kinematics, muscle activity and net muscle moments during gait with different magnitudes and locations of mass added to the legs using a within-subjects design. Participants ( $\mathrm{n}=5$ males) walked on a treadmill with a built in force plate at a constant velocity of $1.25 \mathrm{~m}^{*} \mathrm{~s}^{-1}$. External loads of $0 \mathrm{~kg}, 2 \mathrm{~kg}, 4 \mathrm{~kg}$, and $8 \mathrm{~kg}$ were placed on different parts of the thigh and lower leg, with $16 \mathrm{~kg}$ being placed on the waist of participants. Loads prescribed and the location of loads varied by testing condition following randomization. A strong predictor was found between how distal external mass was placed and the I about the hip for that leg $\left(r^{2}=0.43\right)$ and it was found that net metabolic expenditure during walking increased similarly. Though energy expenditure differed, the study did not find significant differences in muscle moments, activation, or movement kinematics during the swing phase. This suggests that movement efficiency may increase with more distal loading of the lower body without compromising learned movement mechanics or muscle activation patterns during locomotion.

A study by Cavanagh \& Kram (15) had explored the movement kinematic and gait impacts of adding mass to the lower body during distance running within a 
fixed velocity range ( 3.15 to 4.12 miles per hour). The authors also explored the influence of anthropometric factors on these outcome variables, including location of limb mass distribution across the lower body. Participants were accustomed to regular treadmill running and aerobically trained prior to recruitment $\left(\mathrm{VO}_{2 \max }>54 \mathrm{ml}^{*} \mathrm{~kg}^{-1}{ }^{*} \mathrm{~min}^{-1}\right.$. Participants performed a series of running trials on a treadmill at various velocities with external mass $(1.1 \mathrm{~kg})$ present or absent on the lower body. It was observed that the addition of external mass did not alter stride frequency or stride length of participants. It was additionally observed that anthropometric variables such as mass distribution did not significantly influence stride frequency or length. This suggests that a manipulation of mass distribution within the lower body will not result in unfavorable alteration of movement mechanics or kinematics resulting in a decrease in run performance, supporting the practical application of the present study in that movement mechanics may be altered by a redistribution of mass along an axis.

An investigation by Kumagai et al. (6) explored muscle morphology and the influence of associated movement mechanics on sprint performance by crosssectionally evaluating elite male 100 meter sprinters $(n=37$, sprint experience: $7.8 \pm 1.9$ years). Participants underwent measurements of limb length, fat free mass, skeletal muscle distribution, and morphology. It was found that sprinters with faster record times had greater muscle fascicle length in all quadriceps muscle regions and that these lengths which resulted in 
greater proximal muscle thickness were significantly related to record 100 meter sprint performance $(r=0.40$ to 0.57$)$. This suggests that those with longer fascicles and mass distributed proximally with the same amount of overall mass in their thigh will experience greater athletic performance. This may be due to an associated proximal shifting of CoM resulting from the proximal hypertrophy localization these sprinters experienced in past training. This further supports the practical application of the present study in consideration of sports performance.

\section{Localized Atrophy of the Quadriceps Femoris and Treatment}

A systematic review by Giles et al. (25) explored the role quadriceps femoris atrophy takes in patellofemoral syndrome and evaluated the effectiveness of current quadriceps femoris strengthening treatments utilized by physical therapy practitioners. The reviewers' criteria resulted in inclusion of ten other studies and had performed several meta-analyses on various subsets of these studies dependent on outcome variables explored. Quadriceps atrophy was found within the limb affected by patellofemoral pain syndrome $(P=0.036)$, however no significant difference was found in atrophy between the vastus medialis oblique and the vastus lateralis $(P=0.179)$. The review found that quadriceps strengthening of some type would be beneficial in rehabilitating patellofemoral pain syndrome associated quadriceps femoris atrophy. This supports that strengthening the quadriceps femoris is some way may be 
beneficial to managing patellofemoral syndrome, however a limitation of this review is that there was no differentiation between magnitude of improvement encountered by different types of knee extension exercises.

A separate review by Peters \& Tyson (30) investigated differences in location of strengthening along the length of the quadriceps in treating patellofemoral pain syndrome. Randomized controlled trials $(n=3)$, cohort studies $(n=3)$, a clinical controlled trial $(n=1)$, and a case series $(n=1)$ were included in the review. It was found that proximal strengthening of the quadriceps femoris resulted in reduction in pain associated with patellofemoral pain syndrome $(65.1 \pm 22.9 \%)$ and an increase in function involving the patella $(37.5 \pm 37 \%)$. Proximal strengthening was performed by isolating the rectus femoris via hip flexion tasks. The results of this review and the associated meta-analysis suggest that targeted strengthening of specific regions of the quadriceps femoris may facilitate rehabilitation and recovery more than others.

Witvrouw et al. (26) had explored the specific application of OKC and CKC exercises on patellofemoral pain. If these exercises differentially induce hypertrophy localization within the quadriceps femoris, and if proximal strengthening is more rehabilitative than distal, then one should preferentially facilitate patellofemoral pain reduction greater than the other. Participants $(\mathrm{n}=$ 60) were randomly assigned to either an OKC or CKC five week knee extension program. Participants were evaluated for pain associated with their 
condition immediately prior to beginning the intervention, immediately following the intervention, and 3 months after the conclusion of the intervention. It was found that both $\mathrm{OKC}$ and $\mathrm{CKC}$ training resulted in pain relief immediately post intervention and 3 months following the intervention $(P<0.05)$. However, CKC training resulted in greater lower extremity functioning and pain reduction 3 months following the intervention when compared to OKC training. These variables include nighttime pain $(P=0.024)$, frequency of knee locking $(P=$ 0.03), pain during isokinetic testing $(P=0.028)$, and clicking sensations $(P=$ 0.041). The findings of this study suggest that both training methods are effective at treating patellofemoral pain syndrome, but are differentially so, with closed chain being preferential. Longer term effects are not known however.

\section{Inhomogeneity, OKC and CKC Exercises, Movement Mechanics, and Atrophy Conclusion}

There is strong evidence supporting the existence of hypertrophic inhomogeneous muscle morphological changes in the quadriceps femoris induced by exercises involving knee extension joint action. These changes may vary with specific exercise type given relative muscle activation of the quadriceps and its four muscular compartments vary with different hip position and exercise typing. According to previous research, inhomogeneity of hypertrophy appears to occur longitudinally across the length of the quadriceps femoris rather than laterally with localization being preferentially 
proximal or distal depending on exercise condition. This carries implications for movement mechanics and performance given mass distribution along the thigh will shift CoM thereby shifting $I$. This shifting may result in altered movement efficiency from a net energy expenditure standpoint and alter resistance to angular acceleration along with resistance torque encountered during hip flexion tasks, but it is not supported to significantly impact movement kinematics or mechanics in a unfavorable way. Proximal mass distribution and by extension CoM along the thigh have been supported to result in improved performance within elite sprinters supporting the practical importance of hypertrophy localization. It has been additionally supported by numerous reviews and independent studies that atrophy of the quadriceps femoris is related to patellofemoral syndrome and the treatment of localized atrophy can both reduce pain and improve lower extremity function.

Differences have been found between proximal and distal strengthening of the quadriceps femoris and OKC and CKC interventions to treat patellofemoral pain syndrome, suggesting that targeting hypertrophy induction and muscle strengthening to specific regions of the quadriceps femoris via selection of exercise type may facilitate rehabilitation, with proximal strengthening and CKC training supported to be most effective. This supports the present study's assumption of practical application in that adaptation occurs differentially between open and closed chain exercises and that this adaptation results in differently effective treatment for patellofemoral syndrome. 


\section{CHAPTER 3}

\section{METHODOLOGY}

\section{Experimental Design}

The present study was a randomized control trial utilizing a within subjects repeated measures design (See Figure 5). Participants $(n=12)$ were recruited via email outreach campaign, in-class announcements, and local flier using IRB approved recruitment methods and designs. Initially, 15 participants were recruited, however only 12 were able to successfully complete the study and were thus the only participants included in all data and calculations. Of the three participants, one was removed for failure to adhere to study protocols and another two were unable to continue due to reasons outside of the study's control. Participants were allowed to participate upon completing a health history questionnaire and an informed consent document, which participants were required to convey their understanding using the teach-back method. Participants of either gender between the ages of 18 and 35 were recruited. Participants were eligible for inclusion if, within the most recent 6 months, they resistance trained their lower body less than 2 days per week or performed no resistance training at all. If participants were previously regularly performing light to moderate aerobic activity, they were allowed to continue provided no alterations be made to their habits. Participants were excluded if they had any clinical contraindications to lower body exercise or have experienced significant lower body muscle, joint, or tendon injury that may inhibit data 
collection or administration of the training intervention. During the duration of the intervention and data collection periods participants were not allowed to engage in other lower body resistance training activities, start any other new exercise regimen, or begin participating in any new sport. Data collection occurred in the University of Rhode Island Department of Kinesiology BodyComposition Lab, Human Performance Lab, the South County Hospital Diagnostic Imaging Center Lab, and a special lab designed for the current study to perform strength testing and administer the training intervention:

Research lab \#120.

Figure 5. Study Flowchart

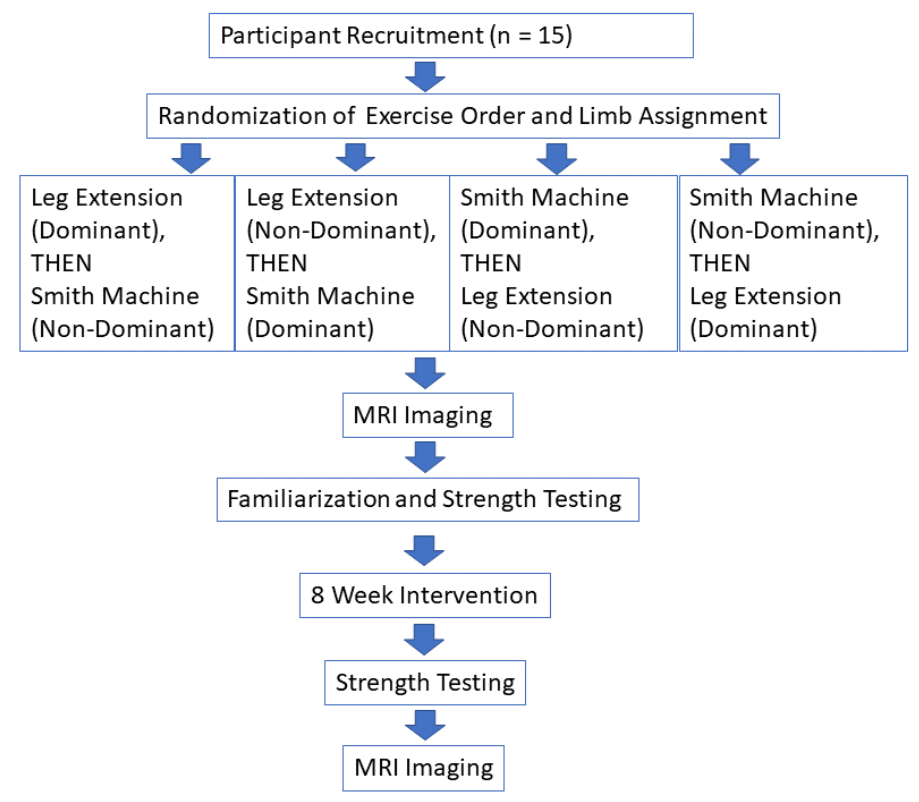

The above figure depicts the design of the present study. Originally 15 participants were recruited, but only 12 completed the entirety of the study. 
Following recruitment, participants underwent MRI imaging of their lower extremities. On a separate occasion, participants then performed an exercise familiarization and muscular strength testing session for the unilateral smith machine back squat and unilateral knee extension. Following this, participants completed a minimum of 21 (maximum of 24) resistance training sessions over an 8 week intervention period where one of the two exercises was assigned to each leg dependent on preconstructed block randomization tables (See Table 1). Resistance training occurred on 3 non-consecutive days per week and periodization was used to facilitate muscular hypertrophy dependent on guidelines set forth by the National Strength and Conditioning Association (31). Following the completion of the intervention, further muscular strength testing and MRI imaging took place, repeating the earlier protocols.

Table 1: Randomization

\begin{tabular}{|c|c|c|}
\hline Participant Number(s) & Limb Assignment & Exercise Order \\
\hline $2,5,12$ & Dominant - Non Dominant & $\begin{array}{c}\text { Closed Chain - } \\
\text { Open Chain }\end{array}$ \\
\hline $1,7,10,13$ & Non Dominant - Dominant & $\begin{array}{c}\text { Closed Chain - } \\
\text { Open Chain }\end{array}$ \\
\hline $3,8,9,14$ & Dominant - Non Dominant & $\begin{array}{c}\text { Open Chain - } \\
\text { Closed Chain }\end{array}$ \\
\hline $4,6,11,15$ & Non Dominant - Dominant & $\begin{array}{c}\text { Open Chain - } \\
\text { Closed Chain }\end{array}$ \\
\hline
\end{tabular}

The above table depicts study randomization, where participants were assigned to performance exercises in a fixed order each training day and with a fixed leg assigned to each exercise. This was done to control for the effects of leg dominance and exercise order. 
The training intervention took place exclusively in a specially set up lab in the Department of Kinesiology at the University of Rhode Island. IRB approval was achieved on 8/23/2019 and data collection occurred between 10/7/19 and $12 / 13 / 19$. Compensation of $\$ 250$ was awarded to participants upon completion of the study, with compensation prorated for study dropouts.

\section{Experimental Descriptors}

Demographics: Participant gender, leg dominance, training status, medication use, and age were assessed via Health History Questionnaire.

Height and Weight: Participant height was assessed via stadiometer (Seca 213, Chino, CA). Participant height was assessed once participants performed an exhalation while standing completely still without wearing shoes (32). Weight and \% body fat were assessed via Bioelectrical Impedance Analysis (InBody 770 scanner, Seoul, Korea) with shoes removed and pockets emptied. Body composition through BIA testing has been supported to be both valid and reliable, utilizing a two component model of body composition (33). The calculated ratio of these two tissues was affected by hydration status when using the BIA device so body composition testing validity was supported by participant hydration status testing $(34,35)$. Hydration status was assessed via refractometer upon collection of a urine sample inserted mid-stream (ATAGO USA, Inc.). Euhydration was defined as having a urine specific 
gravity $\leq 1.025$. If urine specific gravity was greater than this value, participants were asked to consume an appropriate amount of water and urine specific gravity was assessed again every 90 minutes until euhydration status was achieved, in accordance with past validated research practices and the approximate time of the full absorption rate of fluid in the human body (34-36). This had occurred one time and no participants were found to be hyperhydrated. Other controls for body composition analysis such as limiting pre-testing exercise and caffeine consumption were enacted.

\section{Pre and Post Intervention Data Collection Procedure Exercise Familiarization and Technique:}

Prior to the intervention and muscular strength testing, participants were familiarized with each of the exercises they would be performing during the study. Participants began by performing a 2.5 minute warm up on a stationary cycle (Monark 915E, Monark Exercise AB, Sweden) at a cadence of 60 revolutions per minute and resistance of $0.5 \mathrm{kp}$. Intensity, duration, and cadence were selected to facilitate performance and minimize injury while simultaneously minimizing localized and whole body fatigue $(37,38)$. Seat height was set to allow for a $5-15^{\circ}$ bend at the knee when a knee was fully extended on the cycle. Seat height was recorded and replicated in all future sessions. Following this, participants performed the following dynamic stretches for the agonist muscles used in both the unilateral smith machine 
back squat and unilateral leg extension: knee hugs, quad pulls, and leg swing. One set of eight repetitions were performed for each leg for all three stretches. Participants were then familiarized with the unilateral knee extension exercise and machine (Valor Fitness, 2COO41BM CC-4 Leg Machine) as well as the Smith machine that the unilateral back squat was performed on (Body-Solid Powerline, PSM144X Smith Machine). The knee extension machine was adjusted so that participants were positioned in such a way that the lateral epicondyle of their femur was in line with the axis of rotation of the machine. The pad of the machine was oriented on the anterior aspect of the lower limb. Back rest was positioned so that participants' femoral lateral epicondyles were in line with the axis of rotation of the leg extension machine. Consistency in exercise range of motion was ensured by reference gauge (a target string was positioned at the end of range of motion) and range of motion was maintained between $90^{\circ}$ and $180^{\circ}$ of knee extension. The target string was transfixed parallel to the floor between a tripod and nearby wall, which allowed it to be adjusted dependent on the height at which $180^{\circ}$ of knee extension was reached for each respective participant. When participants made contact with the string in a given repetition, $180^{\circ}$ of knee extension was reached. All extension occurred with toes in a neutral position / anterior orientation (See Figure 6)(39). All squatting took place in front of a mirror. Unilateral Smith machine squat technique required participants experience the same range of motion at the knee joint as the unilateral knee extension exercise of $90^{\circ}$ of motion between $90^{\circ}$ and $180^{\circ}$. Reliability between reps was 
ensured via assigned foot position, indicated by tape positioned on the floor. A measuring stick was oriented and fixed on the side of the Smith machine to track and standardize bar displacement with each rep to ensure the appropriate depth of squat was reached consistently. Participants placed the leg assigned to the training condition underneath the hip and immediately anterior to the bar path. Toe angle had a $0^{\circ}$ deviation from the anterior direction (39). Rear foot remained elevated to isolate the quadriceps of the anterior leg (See Figure 7). 
Figure 6. Unilateral Leg Extension

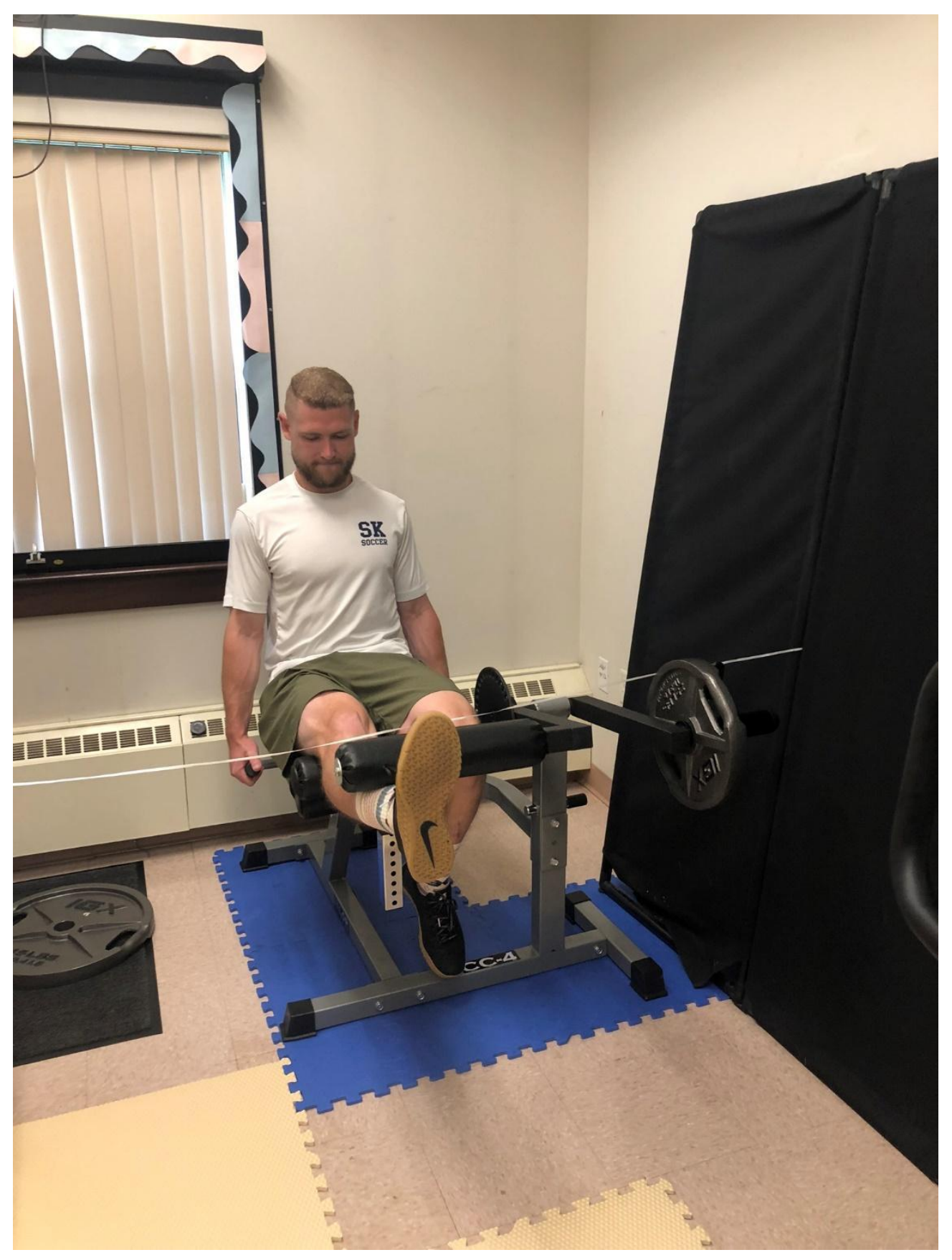

The above figure depicts the OKC movement being performed within the present study. 
Figure 7. Unilateral Squat

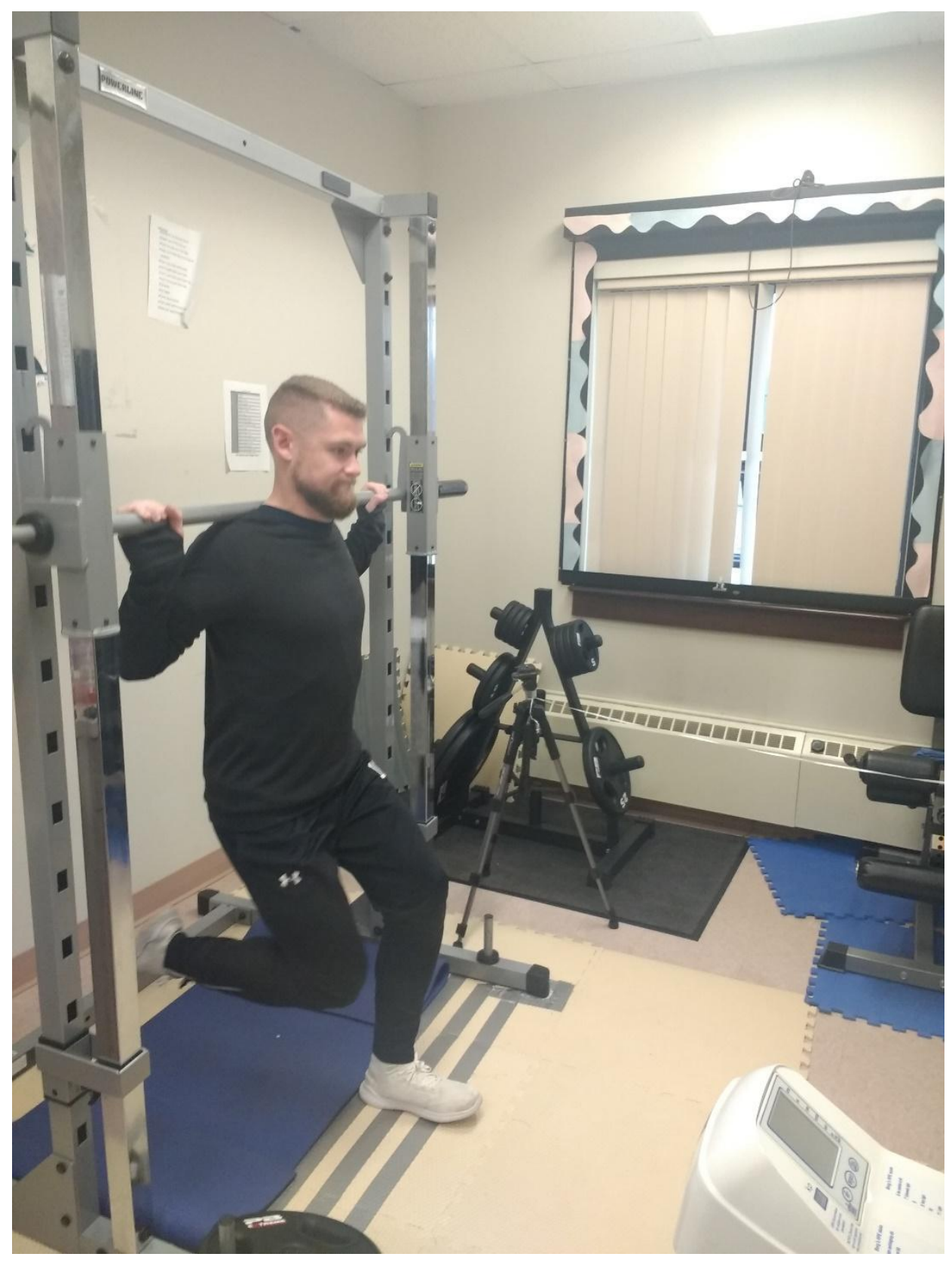

The above figure depicts the CKC movement being performed within the present study. 
Muscular Strength Testing: Participants performed muscular strength testing for each exercise condition on its randomly assigned leg before and after the training intervention (40). Pre-intervention strength testing took place after a familiarization session and MRI session and at a minimum 48 hours prior to the beginning of the first training session. Post-intervention strength testing took place 48-96 hours after the completion of the final intervention training day. Multiple repetition max testing was performed to determine muscular strength and assign training loads as the participant population was not habitually trained and testing parameters more directly carried over to intervention training parameters for repetition prescription. Participants performed a warm up on a stationary cycle for 2.5 minutes at $20 \mathrm{~W}$ of resistance, maintaining a cadence of 60 revolutions per minute, followed by the previously described dynamic warmup. Past research has supported that inclusion of a specific warm up following a general and dynamic warm up increases repetition maximum performance in muscular strength testing (38). Participants then performed 1 warm up set at $50 \%$ of their predicted one repetition maximum in accordance with National Strength and Conditioning Association standards (31). Participants were then provided up to 2 attempts to achieve a 4-12 repetition maximum, which is in accordance with National Strength and Conditioning Association Guidelines (31). After identifying their repetition maximum for a movement, their one repetition maximum was calculated and participants then began a specific warm up for the other 
exercise and repeated the strength testing procedure on the other leg with the untested exercise (See Table 2). The order in which exercises and legs were tested was dependent on the block randomization table participants were assigned to at the start of the study.

Table 2: RM Testing Protocol

\begin{tabular}{|l|l|l|l|}
\hline \multicolumn{1}{|c|}{ Set } & \multicolumn{1}{|c|}{$\begin{array}{c}\text { Estimated } \\
\text { Intensity }\end{array}$} & \multicolumn{1}{|c|}{$\begin{array}{c}\text { Duration / } \\
\text { Repetitions }\end{array}$} & \multicolumn{1}{|c|}{ Rest (min) } \\
\hline Cycling (60rpm) & $0.5 \mathrm{kp}$ & $2.5 \mathrm{~min}$ & N/A \\
\hline Knee Hug & N/A & 8 & N/A \\
\hline Quad Pull & N/A & 8 & N/A \\
\hline Leg Swing & N/A & 8 & N/A \\
\hline Bodyweight Squat* & N/A & 8 & 1 \\
\hline 1 & $50 \%$ & $5-6$ & 1 \\
\hline 2 & $65 \%-90 \%$ & $4-12$ & $2-3$ \\
\hline 3 & $65 \%-90 \%$ & $4-12$ & $2-3$ \\
\hline
\end{tabular}

The above table depicts the multiple repetition maximum protocol used in the present study. Set 2 and 3 were used to determine one repetition maximum. Set 3 was only performed if one repetition maximum could not be determined dependent on set 2 performance.

${ }^{*}$ This warmup exercise was only included in the strength testing day and not included in the training intervention.

MRI Imaging and 3D modelling Open Bore MRI (Magnetom Aera T-1.5, Siemans, USA, 36) was used to capture the whole lower extremity, from $2 \mathrm{~cm}$ 
superior to the greater trochanter of the femur to the most distal portion of the thigh. Thigh length was defined as the distance from the most superior aspect of the femoral head to the most inferior aspect of the femur. This imaging took place once prior to and once following the intervention to assess intervention induced changes in localized hypertrophy and associated parameters. Prior to imaging participants completed a 48 hour diet recall log to support reliability between pre and post intervention MRI scans and image analysis. This recall log was only used to record dietary intake once immediately prior to preintervention MRI testing. Participants then replicated that diet to the best of their ability prior to post-intervention MRI testing to limit the influence of carbohydrate consumption and water retention on MRI results, further isolating the training effect in analysis. Logs were not entered into a nutrient data base and compared. Progressive transverse scans were taken throughout the lower extremities in $1.2 \mathrm{~cm}$ slices. Muscle cross sectional area was measured in the quadriceps femoris within its four compartments (the rectus femoris, vastus lateralis, vastus medialis, and vastus intermedius) along with total thigh length and volume using the OsiriX Dicom Viewer image analysis software. The software was also used to assess compartmental and total muscle cross sectional area of the quadriceps femoris at positions equal to 33\% (proximal), $50 \%$ (middle), and $66 \%$ (distal) of thigh length to allow for calculations of $I$ about the hip via location of thigh CoM (42). To standardize length, clear anatomical landmarks were identified on each set of scans. The first appearance of the femoral head denoted the most proximal aspect of the 
femur, and the portion most immediately superior to the patella denoted the most distal aspect. To account for user identification error, pre intervention and post intervention scans for the same participants were used to ensure consistency in anatomical landmark and muscular compartment identification. Whole quadriceps femoris and individual muscular compartmental volumes were calculated using cubic spline interpolation methods $(11,43)$. These methods mathematically model the cross sectional area and volume of the quadriceps femoris derived from a fixed number of known points with known cross sectional area at those points. The area under the curve of the modeled quadriceps femoris was multiplied by a known mass constant, which allowed the position of CoM to be identified and used in calculating $I$. These values measured CoM and $I$ of the quadriceps and not the entire thigh.

\section{Intervention}

Periodization: The 8 week training period with 3 non-consecutive training days per week involved 6 hypertrophy microcycles and 2 strength microcycles to facilitate the end goal of muscular hypertrophy. Participants were required to complete a minimum of 21 of the 24 training sessions. The acute program variables of intensity, duration (repetitions), volume (sets), and progression were manipulated using the $4 \times 2$ and $2 \times 2$ rules (See Table 3). Initial prescriptions of intensity was dependent on initial one repetition maximums for each exercise as determined by the muscular strength testing protocol. In accordance with the 2 for 2 rule, absolute training loads increased alongside 
increases in participant muscular strength to maintain desired training intensity (31).The training frequency of $3 x$ per week, set and rep ranges, and prescribed intensities reflected recommendations for a novice trainee pursuing hypertrophy during the hypertrophy microcycles and strength during the strength microcycles (See Tables 4, 5, and 6) (31). Participant diet, hydration, and caffeine intake were not tracked during the duration of the 8 week intervention.

Table 3: 8 Week Intervention Periodization

\begin{tabular}{|c|c|c|c|c|c|}
\hline Week & Training Goal & Sets & Reps & Load (\%1RM) & Rest (s) \\
\hline 1 & Hypertrophy & 3 & 12 & 65 & 90 \\
\hline 2 & Hypertrophy & 3 & 10 & 75 & 90 \\
\hline 3 & Hypertrophy & 3 & 8 & 80 & 90 \\
\hline 4 & Strength & 4 & 6 & 85 & 120 \\
\hline 5 & Strength & 4 & 6 & 85 & 120 \\
\hline 6 & Hypertrophy & 3 & 12 & 67 & 90 \\
\hline 7 & Hypertrophy & 3 & 10 & 75 & 90 \\
\hline 8 & Hypertrophy & 3 & 8 & 80 & 90 \\
\hline
\end{tabular}

The above table depicts the exercise prescription used in the present study's 8 week intervention.

Table 4. NSCA Guidelines for Resistance Training Status

\begin{tabular}{|l|l|}
\hline Training Status & Training Experience To Classify \\
\hline Beginner & $<2$ Months \\
\hline Intermediate & $2-6$ Months \\
\hline Advanced & $>12$ Months \\
\hline
\end{tabular}

The above table depicts the NSCA's guidelines for resistance training status of individuals. 
Table 5. NSCA Guidelines for Resistance Training Frequency

\begin{tabular}{|l|l|}
\hline Training Status & Frequency Per Week \\
\hline Beginner & $2-3$ \\
\hline Intermediate & $3-4$ \\
\hline Advanced & $4-7$ \\
\hline
\end{tabular}

The above table depicts the NSCA's guidelines for resistance training frequency.

Table 6. NSCA Guidelines for Resistance Training Targeted Workout Prescription

\begin{tabular}{|l|l|l|l|l|}
\hline Goal & Intensity & Sets & Repetitions & Rest \\
\hline $\begin{array}{l}\text { Muscular } \\
\text { Hypertrophy }\end{array}$ & $67-85 \% 1 \mathrm{RM}$ & $3-6$ & $6-12$ & $30-90$ seconds \\
\hline $\begin{array}{l}\text { Muscular } \\
\text { Strength }\end{array}$ & $\geq 85 \% 1 \mathrm{RM}$ & $2-12$ & $\leq 6$ & $60-300$ seconds \\
\hline
\end{tabular}

The above table depicts the NSCA's guidelines for exercise prescription geared towards specific training goals.

\section{Statistical Approach To The Problem}

Power Analysis: Sample size $(n=15)$ was calculated via an a-priori power analysis using G-Power software, comparing means for matched pairs for a two tailed test. A statistical power of 0.8 and alpha of 0.05 were used. The anticipated effect size of 0.8 (large) was selected following pilot muscle activity data previously presented comparing CKC and OKC exercises. In context of pilot data, an effect size of 0.8 is conservative.

Statistical Analysis: A completers analysis was used rather than an intent to treat analysis given all 3 participants who had dropped out of the study had 
done so within the first 4 weeks and as such it was unlikely adequate time was provided for them to experience a significant hypertrophy effect, especially given the more neurologically focused adaptations during the earliest weeks of training $(31,44)$. Descriptive statistics (mean $\pm S D$ ) were reported to characterize the cohort. Normal distribution was assumed and a condition by time $2 \times 2$ mixed model MANOVA was used to assess the effects of the training intervention on the measured dependent variables of regional muscle cross sectional area, volume, I about the hip, CoM of the thigh, and movementspecific muscular strength. Muscle volume, $I$, and CoM were derived from mathematical modeling and muscular strength was derived from performance on a condition-specific multiple rep max test. The analysis of variance for all dependent variables was conducted between conditions and over time (condition $X$ time). Significant difference was set at an $\alpha$ of 0.05 . MANCOVA was not used to account for confounders given significance was found through the MANOVA with Bonferroni post hoc corrections alone. 


\section{CHAPTER 4}

\section{RESULTS}

\section{Participant Descriptors:}

The 8 week intervention and all associated testing was successfully completed by 12 of the 15 recruited participants (see table 7). Results were not calculated separately by gender given participants acted as their own controls and patterns of hypertrophy should not have been influenced by gender. All participants were considered to have successfully completed the intervention if they attended a minimum of 21 out of 24 scheduled training sessions during the 8 week intervention period. When comparing the legs used for the intervention no differences were observed in leg lengths, as measured via DICOM MRI analysis, between conditions (CKC: $46.67 \mathrm{~cm} \pm 2.36 \mathrm{~cm} \&$ OKC: $46.08 \mathrm{~cm} \pm 2.53 \mathrm{~cm}, \mathrm{p}=0.565$ ) or regional quadriceps femoris volume in the proximal (CKC: $53.21 \mathrm{~cm}^{2} \pm 15.99 \mathrm{~cm}^{2} \&$ OKC: $56.89 \mathrm{~cm}^{2} \pm 26.81 \mathrm{~cm}^{2}, \mathrm{p}=$ 0.365), middle (CKC: $75.98 \mathrm{~cm}^{2} \pm 18.64 \mathrm{~cm}^{2} \&$ OKC: $75.18 \mathrm{~cm}^{2} \pm 20.86$ $\mathrm{cm}^{2}, \mathrm{p}=0.726$ ), or distal (CKC: $61.06 \mathrm{~cm}^{2} \pm 17.87 \mathrm{~cm}^{2} \&$ OKC: $62.89 \mathrm{~cm}^{2} \pm$ $16.16 \mathrm{~cm}^{2}, \mathrm{p}=0.256$ ) locations. 
Table 7. Cohort Characteristics

\begin{tabular}{|l|l|l|l|}
\hline & All $\mathbf{( n = 1 2 )}$ & Male $(\mathbf{n}=\mathbf{7})$ & Female $(\mathbf{n}=\mathbf{5})$ \\
\hline Completed sessions & $23.67 \pm 0.49$ & $23.57 \pm 0.53$ & $23.8 \pm 0.44$ \\
\hline Age (yrs) & $21.25 \pm 3.52$ & $22.28 \pm 4.38$ & $19.8 \pm 0.83$ \\
\hline Height (cm) & $171 \pm 7.60$ & $171 \pm 7.63$ & $163.81 \pm 4.25$ \\
\hline Weight (kg) & $66.46 \pm 9.48$ & $66.46 \pm 9.48$ & $58.63 \pm 6.25$ \\
\hline Body fat (\%) & $18.47 \pm 6.53$ & $14.21 \pm 4.58$ & $24.44 \pm 3.12$ \\
\hline OKC leg length $(\mathrm{cm})$ & $46.08 \pm 2.53$ & $46.71 \pm 2.62$ & $45.2 \pm 2.36$ \\
\hline CKC leg length (cm) & $46.67 \pm 2.36$ & $47.21 \pm 2.967$ & $45.9 \pm 0.89$ \\
\hline
\end{tabular}

The above table depicts characteristics of the cohort of participants that completed the intervention successfully with data pooled and separated by gender.

\section{Strength Assessment:}

Following completion of the intervention, a significant increase in both absolute and relative one repetition maximum (1RM) was observed for both the $\mathrm{CKC}$ (Absolute 1RM: $\Delta 32.39 \mathrm{~kg} \pm 14.48 \mathrm{~kg}, \mathrm{p}<0.001$; Relative 1RM: $\Delta 0.48 \mathrm{x}$ body mass $\pm 0.20 \mathrm{x}$ body mass, $p<0.001$ ) and OKC (Absolute 1RM: $\Delta 17.61$ $\mathrm{kg} \pm 9.74 \mathrm{~kg}, \mathrm{p}<0.001 ;$ Relative $1 \mathrm{RM}: \Delta 0.27 \mathrm{x}$ body mass $\pm 0.14 \mathrm{x}$ body mass, $p<0.001$ ) conditions (Figure 8 ). As the CKC exercise involved muscles both at the knee and hip no direct comparisons were made between 
exercises. However, rating of perceived exertion, as measured via the Borg CR-10 scale of perceived exertion, was similar $(p>0.05)$ between conditions and did not change over time for either CKC $(\Delta 0.83 \pm 2.2, p=0.226)$ or OKC $(\Delta 0.83 \pm 1.4, p=0.845)$ conditions indicating similar exertion between testing
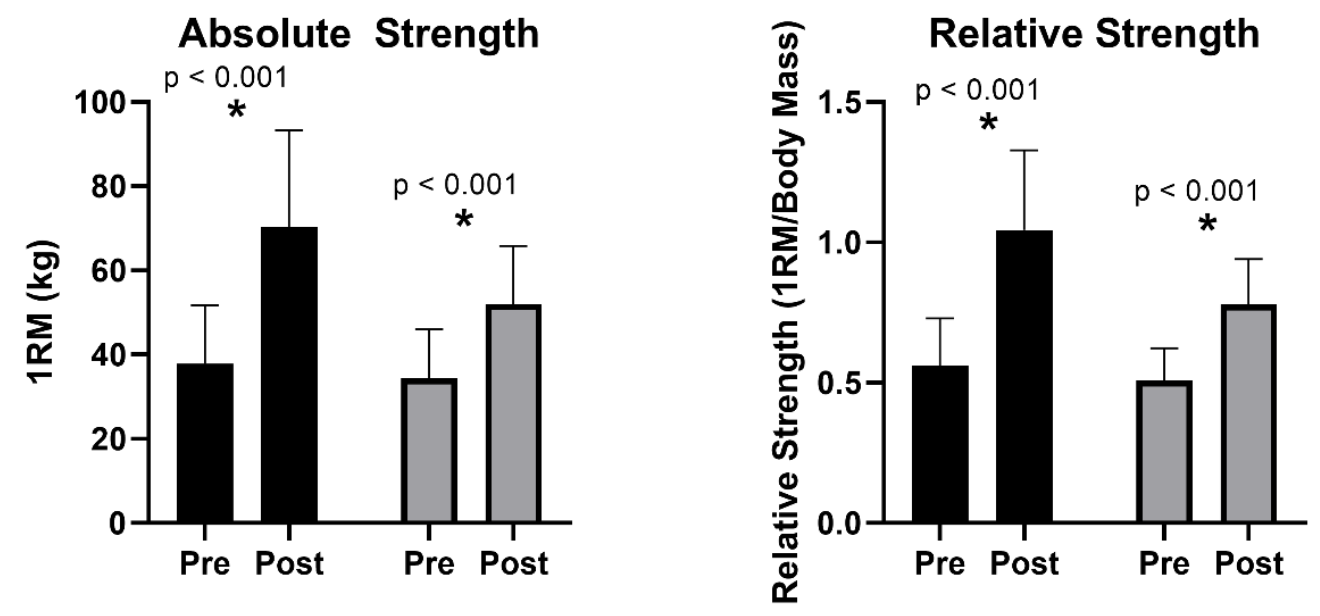

\section{CKC}

口 OKC

Figure 8. Absolute (left) and relative (right) 1RM. Significant differences ( $p \leq$ 0.05) from pre to post intervention are indicated via *.

\section{Muscle Volume \& CSA:}

Whole quadriceps femoris volume significantly increased for both the CKC ( $\Delta$ $\left.60.23 \mathrm{~cm}^{3} \pm 110.52 \mathrm{~cm}^{3}, \mathrm{p}=0.020\right)$ and OKC $\left(\Delta 79.47 \mathrm{~cm}^{3} \pm 87.89 \mathrm{~cm}^{3}, \mathrm{p}=\right.$ 0.020) conditions (Figure 9). Furthermore, the magnitude of increase was similar between conditions for the whole quadriceps femoris $(p=0.285)$ 
indicating that both groups experienced a similar magnitude of hypertrophy (Figure 9).

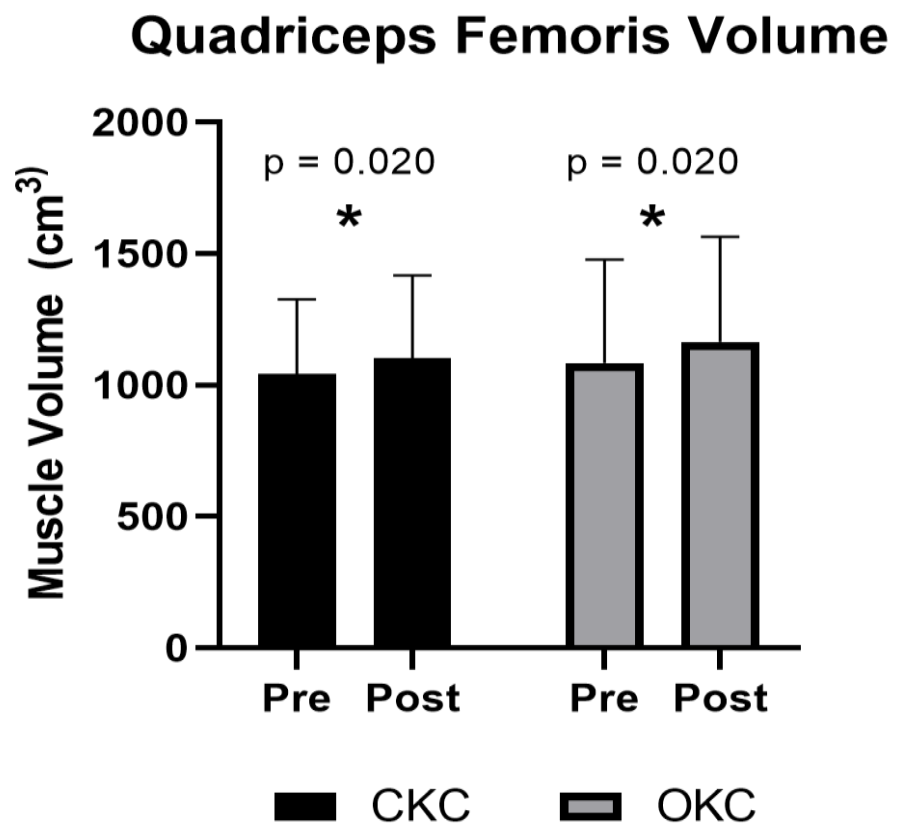

Figure 9. Quadriceps femoris volume. Significant differences $(p \leq 0.05)$ from pre to post intervention are indicated via *.

When comparing changes in quadriceps femoris CSA (CSA) between the 3 regions of interest (proximal-, mid- and distal-thigh), a significant main effect of time was observed for quadriceps femoris CSA change at the middle ( $\Delta 3.572$ $\left.\mathrm{cm}^{2} \pm 1.3 \mathrm{~cm}^{2}, \mathrm{p}=0.020\right)$ and distal $\left(\Delta 7.02 \mathrm{~cm}^{2} \pm 2.45 \mathrm{~cm}^{2}, \mathrm{p}=0.015\right)$ locations, but not proximal $\left(\Delta 0.3 \mathrm{~cm}^{2} \pm 1.43 \mathrm{~cm}^{2}, \mathrm{p}=0.836\right)$ location. Post-hoc analysis revealed that, that the CKC condition experienced a significant increase in CSA at the distal location $\left(\Delta 6.777 \mathrm{~cm}^{2} \pm 2.99 \mathrm{~cm}^{2}, p=0.044\right)$ but not in either the proximal $\left(\Delta 0.3 \mathrm{~cm}^{2} \pm 1.56 \mathrm{~cm}^{2}, \mathrm{p}=0.849\right)$ or middle $(\Delta 1.95$ $\mathrm{cm}^{2} \pm 1.64 \mathrm{~cm}^{2}, \mathrm{p}=0.259$ ) locations (Figure 10). In contrast, the OKC 
condition experienced a significant increase in CSA at both the mid $\left(\Delta 5.2 \mathrm{~cm}^{2}\right.$ $\left.\pm 1.39 \mathrm{~cm}^{2}, \mathrm{p}=0.003\right)$ and distal $\left(\Delta 7.260 \mathrm{~cm}^{2} \pm 2.029 \mathrm{~cm}^{2}, \mathrm{p}=0.004\right)$ locations but not the proximal $\left(\Delta-0.910 \mathrm{~cm}^{2} \pm 1.9 \mathrm{~cm}^{2}, \mathrm{p}=0.643\right)$ location (Figure 10).
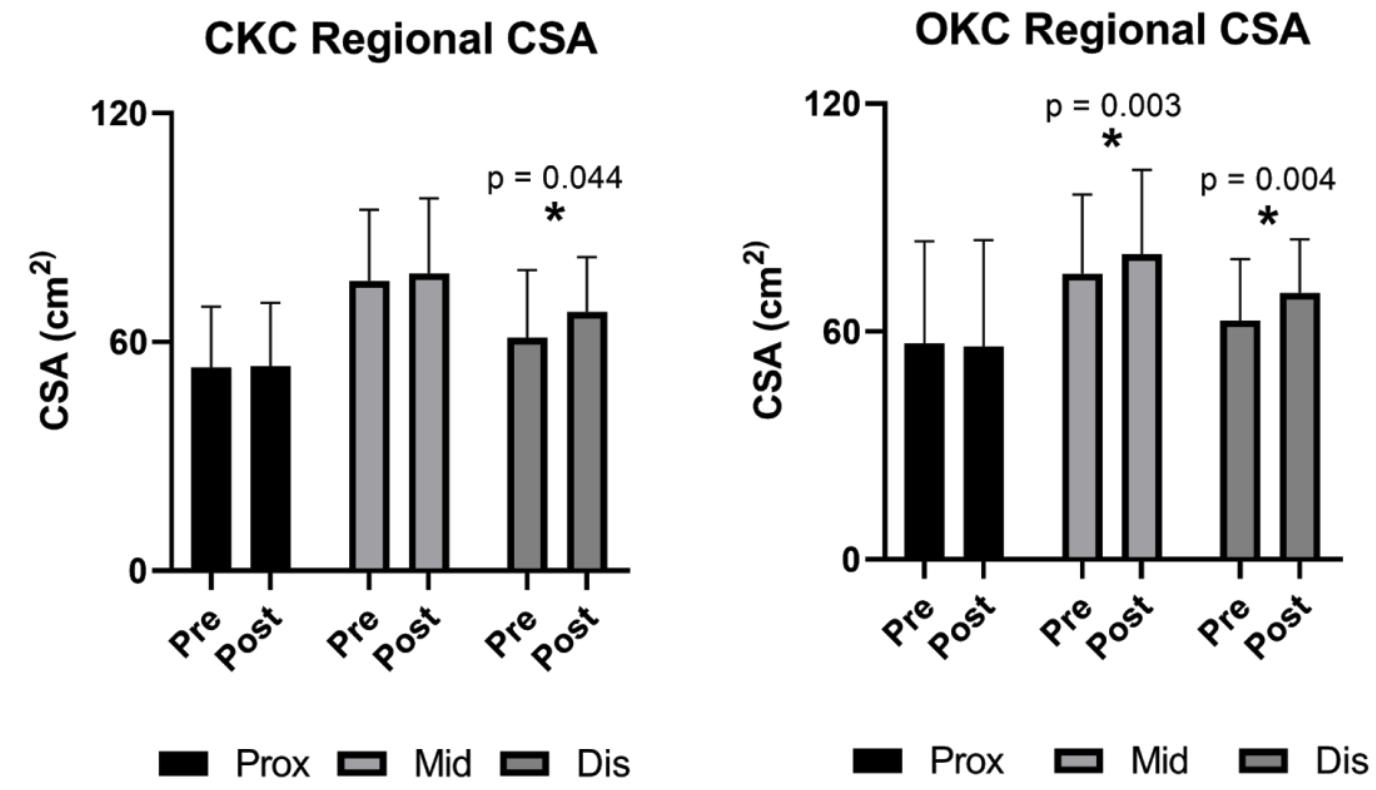

Figure 10. Changes in quadriceps femoris CSA following CKC (left) and OKC (right) training at the proximal (Pros), middle (Mid) and distal (Dis) thigh.

Significant differences $(p \leq 0.05)$ from pre to post intervention are indicated via *.

\section{CoM \& I:}

The location of the CoM of the quadriceps femoris was similar between exercise conditions prior to the exercise intervention $(p=0.457)$. After the exercise intervention the CoM remained unchanged in the CKC exercise condition (Absolute: $\Delta-2.17 \mathrm{~cm} \pm 2.04 \mathrm{~cm}$; Relative: $\Delta-4.67 \%$ leg length \pm 
$4.41 \%$ leg length, $p>0.05$ ) but shifted distally in the OKC condition (Absolute:

$\Delta 1.32 \mathrm{~cm} \pm 1.87 \mathrm{~cm} ;$ Relative: $\Delta 2.81 \%$ leg length $\pm 4 \%$ leg length, $p<$ 0.001) and a significant interaction effect (exercise over time) was observed (Absolute: $\Delta 4.38 \%$ leg length $\pm 0.387 \%$ leg length; Relative: $\Delta 2.41 \mathrm{~cm} \pm$ $0.35 \mathrm{~cm}, \mathrm{p}<0.001$ : Figure 11)

Accompanying changes in location of CoM, similar changes in / were observed as an interaction effect of exercise over time was observed $(\Delta 0.022$ $\left.\mathrm{kgm}^{2} \pm 0.003 \mathrm{kgm}^{2}, \mathrm{p}<0.001\right)$ and $/$ was increased in the OKC condition $(\Delta$ $\left.0.017 \mathrm{kgm}^{2} \pm 0.014 \mathrm{kgm}^{2}, \mathrm{p}<0.001\right)$ but remained unchanged in the CKC condition $\left(\Delta-0.022 \mathrm{kgm}^{2} \pm 0.020 \mathrm{kgm}^{2}, \mathrm{p}>0.05\right.$ : Figure 11).
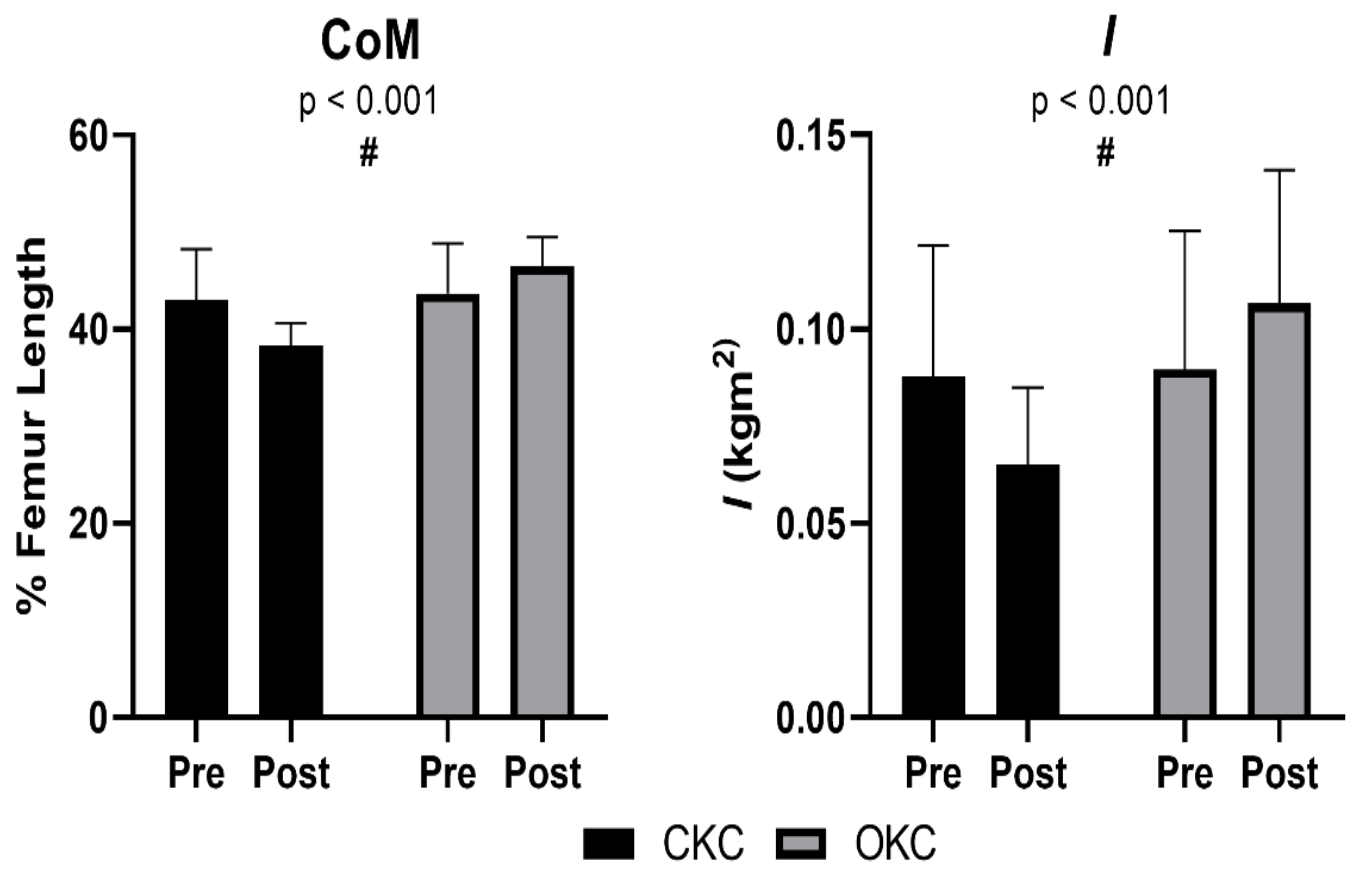

Figure 11. CoM (left) and I (right). Significant differences $(p \leq 0.05)$ in change from pre to post intervention between conditions are indicated via \#. 


\section{CHAPTER 5}

\section{DISCUSSION}

The present study was the first to investigate the differing effects of CKC and

OKC knee extension exercises on patterns of quadriceps femoris hypertrophy and their effect on CoM and I of the muscle relative to the hip. Our findings indicated that patterns of hypertrophy differed between CKC and OKC knee extension exercises and that these patterns differentially shifted CoM and thus differentially altered I about the hip. The OKC knee extension training resulted in an increase in quadriceps femoris CSA both at the middle of the femur and the distal aspect, while the CKC knee extension training only resulted in an increase in quadriceps femoris CSA at the distal aspect. This resulted in a more proximal bias in mass allocation following CKC training than OKC training and thus caused the CoM of the thigh to shift proximally while reducing / about the hip, supporting our hypothesis. Given proximal hypertrophy is beneficial to performance during running as the thigh moves about the hip $(15,45,46)$, these findings suggest that running athletes should skew exercise selection towards CKC knee extensions exercises over OKC, while making these decisions in conjunction with other best standards of practice for exercise prescription and within necessary training parameters. These findings are important as they carry implications for performance and can be used to inform exercise prescription for specific sports performance needs. 
The present study had initially recruited 15 untrained participants and 3 had failed to complete the study. Of the original 15 recruited, selection bias may have occurred given a majority of recruitment had taken place via word of mouth in Kinesiology classrooms. However, all participants were untrained and not all participants were recruited via this method. Of the participants that reached out to the research team, those invited were randomly invited to be screened and if they met study criteria they were recruited. It stands to reason that selection bias would not have altered the results of the study given training status was controlled for and there are no reasonable physiologic differences between the quadriceps of untrained Kinesiology students and untrained students of other majors. Of the 3 participants who had been removed from the study, the bias in removal was arbitrary and unique to each case and should not have skewed the remaining population's data in any way.

It is unlikely that patterns of hypertrophy would have manifested differently in a trained population. Mechanics of OKC and CKC movements do not vary based on an individual's training status so would be expected to elicit similar results if performed within the context of a larger resistance training program. However, those who are well trained adapt at a slower rate than individuals who are untrained. Additionally, athletes who are very well adapted may experience a detraining effect if they are limited to training one movement several times per week. Due to this, repeating the present study in an 8 week 
period with an athletic population may not convey the same clinical significance as the present study and may suggest unfavorable changes in muscle volume and strength. However, if a part of a larger comprehensive program that is maintained habitually athletes should experience similar benefits to the population tested over time. Additionally, at an elite level slight changes in performance make clinically significant impact on sports performance and the exponential effect CoM has on I supports the benefit for athletic populations, even if the total shifting is minimal due to resistance to hypertrophy.

The present study's findings suggest that exercise selection can influence training outcomes in meaningful ways, which may mean that resistance training program design practices should account for exercise selection in ways that have not been previously recommended. Doing so will allow more precise control over the resulting adaptations from training and thereby improve resistance training efficacy. However, more research is needed to support specific programming recommendations within a comprehensive resistance training program.

Patterns of Hypertrophy: Following completion of the 8 week training intervention, patterns of hypertrophy differed between conditions. Patterns of hypertrophy for both exercises were inhomogeneous with proximal, middle, and distal aspects of the quadriceps femoris undergoing differing degrees of 
hypertrophy. The CKC training only resulted in significant distal hypertrophy while the OKC condition resulted in significant hypertrophy at both the mid and distal quadriceps femoris. Differences in patterns of hypertrophy may be attributed to task specific motor unit recruitment favoring mechanically favorable muscle fiber activation during one condition over the other, though limited research has been conducted on task specific motor unit recruitment to date (24).

Past work by Blazevich et al. (2006) supports that muscular compartments of the quadriceps femoris differ architecturally along their length and thus have mechanically different regional properties regarding their force transmission potential (3). This has been supported to be partly attributable to differences in muscle thickness, which positively relate to differences in muscle fascicle angle (3). Past research by Mitchell et al. (1997) had provided additional support for the existence of the regional differences noted by Blazevich et al. (2006) across the length of muscular compartments of the quadriceps femoris by specifically investigating the vastus medialis (3, 27). Mitchell et al. (1997) identified mechanical differences within the vastus medialis muscular compartment's proximal and distal aspects which suggests that withincompartment muscle fiber recruitment may have been task specific in the vastus medialis $(24,27)$. Given motor unit recruitment has been demonstrated to be task specific and that architectural differences along the length of the individual muscular compartments of the quadriceps femoris have been 
demonstrated to be mechanically dissimilar, it is reasonable to conclude that recruitment within the quadriceps femoris specifically may be task specific. $\mathrm{CKC}$ and $\mathrm{OKC}$ exercises are discrete tasks which place different demands on the body and thus may have resulted in differences in motor unit recruitment. Differences in muscular compartment involvement, quadriceps morphology at the point of peak resistance torque, and differences in motion at the hip joint may have driven this $(3,10,18)$. The greatest muscular hypertrophy has been reported to occur in recruited motor units and active tissue, with magnitude of hypertrophy varying by specific motor unit recruited, thus explaining the differential hypertrophy localization between CKC and OKC conditions reported in the present study (47). Due to co-contraction of other agonists to drive hip extension during the CKC movement that was not present in the OKC movement, the absolute load used for the CKC movement was greater than the OKC movement. However, the relative loading of the quadriceps femoris itself should not have differed between conditions given its force production capacity would not have been altered with the involvement of other muscles, and by extension relative hypertrophy should not have differed dependent on loading (48). The effects on patterns of hypertrophy of these CKC and OKC knee extension movements should reasonably translate to other variations of CKC and OKC knee extension movements.

The quadriceps femoris may have experienced differences in task specific demands, and thus hypertrophy localization, between performing CKC and 
OKC exercises due to inherent differences between exercise type. CKC exercises result in peak torque on the knee joint being reached when the knee is maximally flexed and the quadriceps are maximally lengthened (19). However, OKC exercises result in peak torque on the knee joint being reached when the knee is maximally extended and the quadriceps are maximally shortened (20). Given fascicle angle changes with muscle length, peak torque: a meaningful driver of hypertrophy, was applied to the quadriceps femoris under mechanically different conditions between exercises (47). Fiber angle has been reported to alter muscle fiber force and shortening velocity, thus imposing different mechanical demands on the tissue (29). Furthermore, active muscle tissue has been reported to variably gear, altering muscle fiber angle dependent on task-specific demands to best meet those demands (29). Differences in fascicle length and angle have been demonstrated to have implications for sports performance and facilitate performance differentially in sprinters and runners $(6,22)$. It is reasonable that differences in torque-related demands on the knee joint resulted in differences in muscle gearing and thus different localized hypertrophy responses dependent on the regions that were more suited to the gearing required to meet task demands. However, more research is needed to investigate this.

Differences in patterns of hypertrophy may have also been driven by differences in rectus femoris involvement and subsequent hypertrophy. Though rectus femoris hypertrophy did not significantly differ between the 
CKC and OKC condition ( $p=0.376)$, it may have had a greater role in extending the knee during the $\mathrm{CKC}$ exercise, which is in line with past research due to its biarticular nature (13). This may have partially shifted emphasis away from the other compartments of the quadriceps femoris during training and in having done so reduced the overall amount of hypertrophy in those compartments due to differences in muscle activation $(17,49)$. Those compartments may have been disproportionately responsible for mid-thigh hypertrophy, explaining the lack of significant hypertrophy in that region following the CKC intervention, but more research is needed to conclude this. Thus, the middle of the thigh may have not received as much of a hypertrophy stimulus in the CKC condition.

CoM and I: Significant hypertrophy was observed at two regions distal to this point in the OKC condition and one region beyond this point in the $\mathrm{CKC}$ condition, which resulted in CoM being significantly shifted more distally in response to OKC training. Though non-significant proximal hypertrophy was observed in the CKC condition, the non-significant change was large enough to induce a significant proximal shift in CoM. Given the location of CoM has an exponentially greater impact on $I$ than mass as $I=m r^{2}$, this shift resulted in significantly increased / about the hip in the OKC condition and reduced I about the hip in the CKC condition $(8,10,16)$. The significant difference in I about the hip between conditions suggests that resistance torque of the thigh is lowered and any given amount of angular acceleration about the hip will 
occur with less muscle force following CKC training, and OKC training would result in the opposite $(50,51)$. These changes were measured in the quadriceps exclusively and not the thigh in its entirety because differences in patterns of hypertrophy were expected in the quadriceps specifically while the mass of other aspects of the thigh such as the femur were not expected to meaningfully change in response to the training intervention. However, it stands to reason that a shift in CoM and $I$ in the quadriceps would result in a shift in the thigh itself given the quadriceps muscle is positioned along the longitudinal axis of the thigh shank (4). Though, the effect of the observed patterns of hypertrophy on the CoM and I of the whole thigh is lesser, but still clinically meaningful, than that of the quadriceps alone because the whole thigh is heavier.

Muscular Strength and Size: Despite significance being found in change in muscular size and strength, large standard deviations were observed due to the participant population being comprised of various genders and individuals of varied heights. Absolute muscular strength was reported to have increased for both conditions, supporting that both conditions may benefit sports performance, given greater absolute muscular force production is associated with greater performance in many common sports activities such as running and jumping $(52,53)$. Differences in muscular strength change between conditions were not assessed given the inherent differences in absolute load between the $\mathrm{CKC}$ and $\mathrm{OKC}$ conditions. During the $\mathrm{CKC}$ condition coactivation 
of the gluteus maximus contributes to muscular force production and increases the absolute load used at a given relative intensity contrasted to the OKC condition where the quadriceps femoris is the only large agonist involved in muscular force production. RPE during muscular strength testing was similar from pre to post intervention for the CKC and OKC conditions, suggesting that the strength tests were reliable measures for participants and that the technical difficulty of either movement did not limit pre intervention strength testing performance. Similar reported RPE from pre to post intervention also suggests that perceptions of exercise intensity and ability to exert force were not altered by changes in menstruation status, which have been previously linked to RPE (54). Given the duration of the 8 week intervention period, female participants were likely to have undergone strength testing in similar menstrual cycle phases if their cycles were of a normal length (55). However, given that significant increases in muscular strength and volume from pre to post intervention were observed in both conditions it is reasonably supported that both training conditions result in favorable adaptation in that CKC and OKC training both result in the development of more muscle mass and increased muscular strength. Though increased muscle mass makes a limb harder to move around a joint due to effectively increasing $l$, increasing its size can facilitate greater muscle force production and increase muscular strength, facilitating high performance $(52,53,56)$. Coupling this increase in mass with a favorable shift in CoM, the increase in I about a joint caused by increased mass can be negated by a decrease in I 
and performance predicated on movement around that joint may have meaningfully improved.

Performance and Therapeutic Applications: Given both conditions resulted in favorable adaptation in terms of muscular strength and size, difference in training efficacy was defined by shift in CoM and the resulting change in I. Thus it is supported that CKC training is more beneficial for performance than OKC training during movements where the thigh moves about the hip, such as the swing phase of running, which has been demonstrated to be an important movement in sports performance $(45,57)$. In movements requiring the thigh to move about the knee, OKC training would be more beneficial for performance. It is favorable for athletes to require less muscle force to achieve any given angular acceleration around the hip joint as well as increase peak angular acceleration around the hip joint during a maximal power contraction of the quadriceps femoris $(11,22,58,59)$. Reducing the muscle force required to achieve a given angular acceleration $(\mathrm{T}=l \mathrm{a}$, where $\mathrm{T}=$ effort torque, $I=I$ about the origin, and $a=$ angular acceleration) of the thigh about the hip is beneficial given it will reduce muscular fatigue and prolong the duration of high performance (59). Increasing peak angular acceleration is beneficial in many sports given greater angular acceleration is associated with greater propulsion which increases linear velocity of the whole body and greater linear velocity of the whole body is associated with greater success in many sports $(7,60-62)$. 
The current study supports the use of CKC knee extension movements for athletes, which has been supported by the literature for reasons other than those presented in this study, such as strengthening multiple muscles involved in running action as opposed to just one $(57,64-66)$. Due to this, the current work can be used to inform evidence-based practice and strengthen support for and use of CKC knee extension movements over OKC as it supports current best practices. However, optimal exercise prescription within the context of a full periodized program with appropriate volume for a highly trained athlete is currently unknown. Due to this, skewing training towards prescription of CKC knee extension exercises can be recommended for evidence-based training, however exact prescription parameters are currently unknown.

Therapeutic applications of this research are promising but will vary and require further investigation. Given exercise selection has been suggested to influence hypertrophy localization, targeting hypertrophy induction in injured tissues in specific regions of the quadriceps may be possible and allow for case-specific and condition-specific rehabilitation practices $(25,67)$. Those with general atrophy of the quadriceps femoris may benefit from CKC training as it would facilitate ease of activities of daily living such as standing from sitting and walking by making it easier for movement of the thigh about the hip $(46,68)$. However, this would require further investigation to confirm. 
These results are also informative and useful for the non-athletic general population, as improving / results in an improved running economy, and thus can increase duration of aerobic activity, which can contribute to helping regular individuals meet physical activity guidelines for aerobic exercise more readily $(9,63)$.

Limitations: The present study had multiple limitations. Range of motion for both exercises was fixed to the range of 90 to 180 degrees of knee extension which does not comprise the entire range of motion of knee extension activities of 70 to 180 degrees of knee extension. Due to mechanical limitations in our method of performing a single legged squat, most participants were unable to travel further than 90 degrees without resting their back leg on the floor. If a fuller range of motion were used it is plausible that the magnitude of hypertrophy and thereby effect of the training protocol may have been greater in both conditions due to increased time under tension (47). Due to logistical limitations and time constraints, MRI slices were analyzed at fixed points along the length of the femur and cubic spline interpolation was used to model the rest of the quadriceps femoris derived from the analyzed slices CSA, and their location. Though this method has previously been used by other researchers to model the quadriceps femoris, having every slice analyzed may have allowed for a more comprehensive understanding of differences in hypertrophy localization between conditions $(11,43)$. 
During the duration of the intervention period, protein consumption habits were not tracked for any participants. However, participant protein consumption habits may have influenced results. Given underconsumption of protein has been linked with lesser hypertrophy following resistance training and high consumption has been linked with increases in muscular hypertrophy and strength, it is plausible that the magnitude of change in muscle volume and strength may have differed based on participant consumption patterns (69, 70). This may have resulted in either a blunted observed effect of the intervention. Of additional note, the cohort of participants involved in the study did not have any unusual characteristics that would limit generalizability of the present study's findings.

Conclusion: The present study was the first to investigate differences in regional hypertrophy of a muscle following CKC and OKC training. An untrained population was used, but results are generalizable to larger athletic populations. Both training interventions had resulted in significant and similar hypertrophy of the quadriceps femoris and significant increases in muscular strength. However, localization of hypertrophy varied with CKC training producing significantly distal hypertrophy and OKC training producing significant hypertrophy at the middle and distal aspects of the quadriceps femoris. This resulted in a CoM being shifted distally following OKC training when compared to CKC training and a greater increase in I about the hip after OKC compared to CKC. As a more proximal CoM and lower / provides a 
mechanical advantage when running, and faster sprinters have been observed to have more funnel shaped thighs (greater proximal muscle mass and less distal muscle mass) these results suggest the CKC exercises are more favorable to choose over OKC for runners and athletes who could benefit from faster running speeds or improved running economy. These findings should translate from unilateral $\mathrm{CKC}$ and $\mathrm{OKC}$ movements to bilateral $\mathrm{CKC}$ and $\mathrm{OKC}$ movements when put into practice in the field. Though current understanding of the effects on athletic performance are derived from simple mathematical modeling and not direct observation, therefore further investigations into the direct impact that exercise selection has on patterns of quadriceps hypertrophy and subsequently on athletic performance is required. 


\section{APPENDIX I}

\section{Consent Form for Research}

We hope that you consider taking part in our study examining how exercise affects quadriceps muscle growth, shape, blood flow, and functional performance. We believe that this study (detailed below) has the potentially to significantly improve the effectiveness of sports medicine, athletic, and physical therapy programming by providing important knowledge on what specific types of exercises do to the quadriceps.

STUDY TITLE- Effects of open and closed kinetic chain exercises on proximal vs. distal hypertrophy of the quadriceps.

PRINCIPAL INVESTIGATOR

Principal Investigator: Jacob Earp, Ph.D. Office (401) 874-7845 Email: jacob_earp@uri.edu

\section{KEY INFORMATION}

Important information to know about this research study: 
- The purpose of the study is to determine if the exercises a person uses to train causes their muscle to hypertrophy (grow in thickness) at different locations.

- If you choose to participate, you will be asked to take part in 8 weeks of resistance training in which you will train using the squat exercise on one leg and the leg extension exercise on the other leg for. You'll be asked to train 3 days per week and each training session should last $\sim 30 \min$.

- In addition to the training you will also be asked to take part in 2 days of testing before and after the training. As part of this testing you will have an MRI scan (imaging) of your legs taken at South County Hospital and be asked to perform a strength test.

- The total time commitment to take part in this study is approximately 13.5 hours.

- Risks or discomforts from this research include mild muscle soreness from performing the leg extension exercises.

- The study will be used to determine what aspects of a resistance training or physical therapy program should be emphasized to promote growth in different regions of the quadriceps (a muscle group in your upper leg). This can help people to target their training for specific parts of the muscle which are 1) injured, 2) at risk of injury or 3) important for sport performance.

- You will be provided a copy of this consent form. 
- Taking part in this research project is voluntary. You don't have to participate and you can stop it any time.

\section{INVITATION}

You are invited to take part in this research study. The information in this form is meant to help you decide whether or not to participate. If you have any questions, please ask.

\section{Why are you being asked to be in this research study?}

You are being asked to be in this study because you may be interested in participating in research related to kinesiology, physical therapy or sports medicine. To take part you must be between the ages of 18-35 and currently free from any current injury or illness or any other lower leg injury which might prevent you from being able to safely perform leg extension or squat exercises. Additionally you must not have engaged in regular resistance training exercise (using weights for your lower body 2 or more days per week) for your lower body within the last 6 months.

\section{What is the reason for doing this research study?}


The way in which certain types of exercises (open and closed kinetic chain) affect the way the quadriceps muscle grows and its mechanics are not yet fully known. There is reason to believe that regions of the quadriceps will grow differently depending on whether open and closed chain exercises are performed. If the way that these exercises influence quadriceps growth becomes known then practitioners (in both physical therapy and strength and conditioning) will be better able to design programs for their respective patients and clients. Specific parts of the quadriceps that need to be strengthened and rehabilitated by therapists can be more efficiently targeted, and coaches can train their athlete's quadriceps to better optimize performance.

\section{What will be done during this research study?}

After signing this informed consent document we will ask you to complete a health history questionnaire and physical activity survey to ensure that you are free from any lower body injury, which might interfere with your ability to take part in testing and should your testing session and provide descriptive information (this should take about $10 \mathrm{~min}$ ).

During the pre-training testing, you'll be met at the South County Hospital to undergo a lower body MRI scan. Before undergoing MRI testing you'll complete a food recall log where you'll have to recall what you'd eaten over 
the past day to the best of your ability from memory. You will additionally be asked to provide a urine sample in a specimen collection cup to be analyzed and then immediately discarded before undergoing body composition testing of your entire body via BIA, which is a noninvasive measurement tool that simply requires you stand on a scale. Afterwards you'll perform a standardized warm-up consisting of $5 \mathrm{~min}$ of low intensity aerobic exercise followed by a series of low intensity lower body exercises \& stretches. Afterwards, a small probe will be placed on the skin over your quadriceps muscle that will record muscle activity. Once this set-up is completed you'll be asked to perform a series of two different types of leg exercises. One will be a single leg squat and the other a single leg leg extension. Afterwards, you will be asked to participate in 8 weeks of resistance training (3 days per week), in which you'll perform, these two different exercises on different legs. Once the intervention concludes you will undergo one final MRI scan. The entire study should take approximately 9 weeks and approximately 10.5 hours.

\section{How will my data be used?}

Your data will coded so that you cannot be identified and results from analysis of your data will 
presented at scientific conferences and published in scientific journal without any individual identifiers.

\section{What are the possible risks of being in this research study?}

There are minimal risks to you from being in this research study such as delayed muscle soreness from exercise or mild skin agitation from adhesives used to secure equipment to your skin. There are risks associated with MRI use, however the risks of MRI testing will be minimized via screening, however MRI use is contraindicated if pregnant or with certain other implantations or conditions. To ensure you are eligible to undergo MRI testing a pre-screening form will be administered to you.

\section{What are the possible benefits to you?}

You may experience increased muscle size and strength of your quadriceps muscles on both legs, as would be expected during an 8 week training intervention.

What are the possible benefits to other people? 
The results from this study will provide information that can potentially be used to improve the effectiveness of exercise programs that are designed to help people to prevent or recover from tendon injury.

\section{What are the alternatives to being in this research study?}

Instead of being in this research study you can decide not to take part in this study without any repercussions.

\section{What will being in this research study cost you?}

There is no cost to you to be in this research study.

Will you be compensated for being in this research study?

You will receive $\$ 250$ for the time commitment associated with the study. Several payments will be made during the duration of the intervention to equal this amount for your time investment.

What should you do if you have a problem during this research study?

Your welfare is the major concern of every member of the research team. If you have a problem as a direct result of being in this study, you should 
immediately contact one of the people listed at the beginning of this consent form.

\section{How will information about you be protected?}

Reasonable steps will be taken to protect your privacy and the confidentiality of your study data. The data will be stored electronically through a secure server and will only be seen by the research team during the study. The only persons who will have access to your research records are the study personnel, the Institutional Review Board (IRB), and any other person, agency, or sponsor as required by law. The information from this study may be published in scientific journals or presented at scientific meetings but the data will be reported as group or summarized data and your identity will be kept strictly confidential.

\section{What are your rights as a research subject?}

You may ask any questions concerning this research and have those questions answered before agreeing to participate in or during the study.

For study related questions, please contact the investigator(s) listed at the beginning of this form. 
For questions concerning your rights or complaints about the research contact the Institutional Review Board (IRB) or Vice President for Research and Economic Development:

- IRB: (401) 874-4328 / researchintegrity@etal.uri.edu.

- Vice President for Research and Economic Development: at (401) 874-4576

\title{
What will happen if you decide not to be in this research study or decide to stop participating once you start?
}

\author{
You can decide not to be in this research study, or you can stop being in \\ this research study ("withdraw') at any time before, during, or after the \\ research begins for any reason. Deciding not to be in this research study or \\ deciding to withdraw will not affect your relationship with the investigator or \\ with the University of Rhode Island (list others as applicable).
}

You will not lose any benefits to which you are entitled.

\section{Documentation of informed consent}

You are voluntarily making a decision whether or not to be in this research study. Signing this form means that (1) you have read and understood this 
consent form, (2) you have had the consent form explained to you, (3) you have had your questions answered and (4) you have decided to be in the research study. You will be given a copy of this consent form to keep.

\section{Participant Name:}

(Name of Participant: Please print)

\section{Participant Signature:}

Signature of Research Participant

Date

Investigator certification:

My signature certifies that all elements of informed consent described on this consent form have been explained fully to the subject. In my judgment, 
the participant possesses the capacity to give informed consent to participate in this research and is voluntarily and knowingly giving informed consent to participate.

Signature of Person Obtaining Consent

Date 


\section{APPENDIX II}

\section{Health History Questionnaire}

HEALTH HISTORY QUESTIONNAIRE

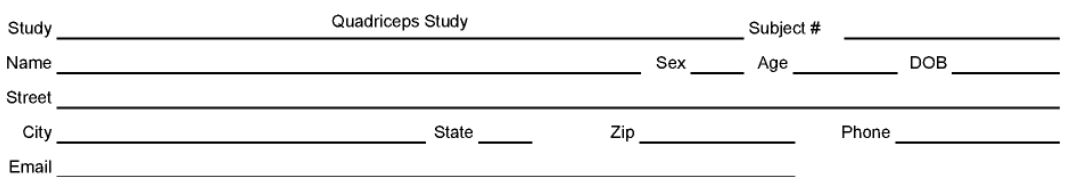

PLEASE ANSWER ALL OF THE FOLLOWING QUESTIONS AND PROVIDE DETAILS FOR ALL "YES" ANSWERS IN THE SPACES AT THE BOTTOM OF THE FORM.

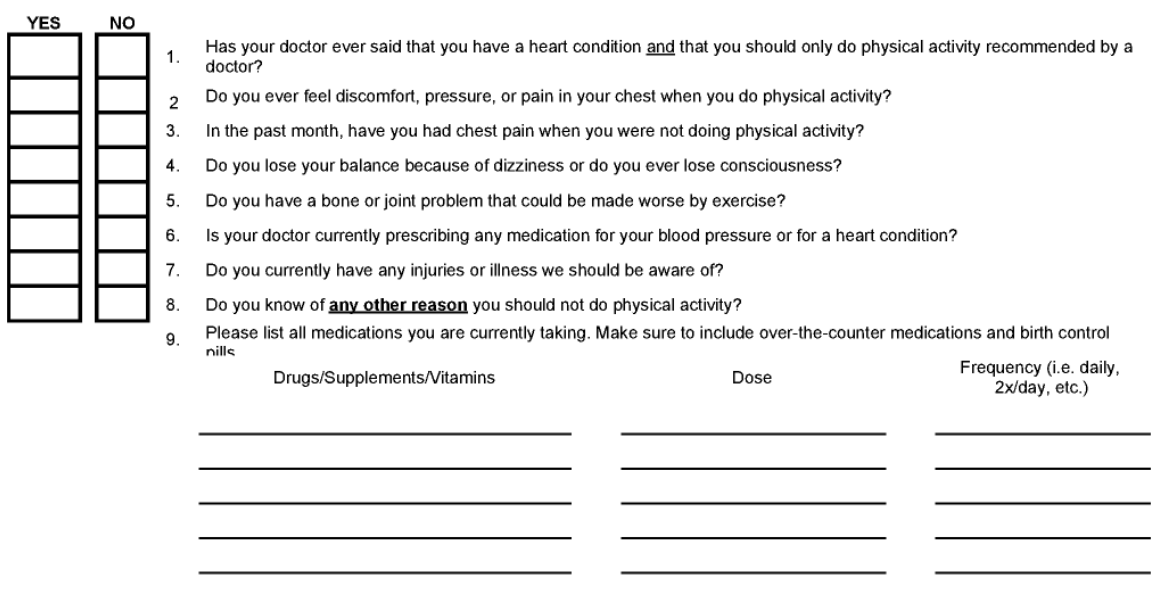

10. Please check the box next to any of the following body parts you have injured in the past and provide details
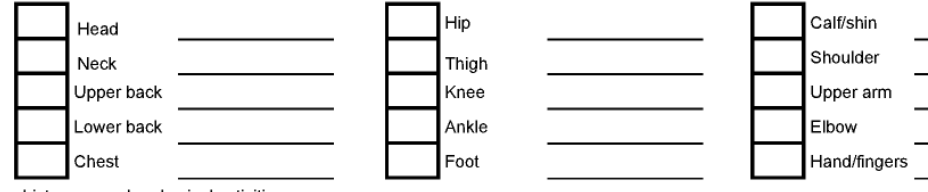

11. List your regular physical activities

How often do you do it?

How long do you do it?

How long ago did yo
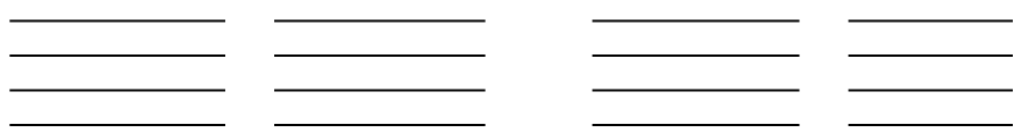

ADDITIONAL IF YOU SELECTED YES TO ANY OF THE QUESTION ABOVE PLEASE PROVIDES DTAILS (WHAT HAPPENED AND DETAILS: 


\section{APPENDIX III}

\section{Two Day Food Record}

Write down everything you ate yesterday. Include all meals and snacks and the amount eaten.

Day 1

\begin{tabular}{|l|l|l|l|l|}
\hline Meal \& & Food or & Amount & Method of & Do not write in \\
Time & Beverage & & Preparation & this space \\
\hline Breakfast & & & & \\
\hline & & & & \\
\hline Snack & & & & \\
\hline & & & & \\
\hline Lunch & & & & \\
\hline & & & & \\
\hline Snack & & & & \\
\hline & & & & \\
\hline & & & & \\
\hline & & & & \\
\hline & & & & \\
\hline & & & & \\
\hline & & & & \\
\hline & & & & \\
\hline & & & & \\
\hline & & & & \\
\hline & & & & \\
\hline & & & & \\
\hline & & & & \\
\hline & & & & \\
\hline & & & & \\
\hline
\end{tabular}




\begin{tabular}{|l|l|l|l|l|}
\hline & & & & \\
\hline & & & & \\
\hline Evening & & & & \\
\hline & & & & \\
\hline & & & & \\
\hline
\end{tabular}

\section{Day 2}

\begin{tabular}{|l|l|l|l|l|}
\hline Meal \& & Food or & Amount & Method of & Do not write in \\
Time & Beverage & & Preparation & this space \\
\hline Breakfast & & & & \\
\hline & & & & \\
\hline Snack & & & & \\
\hline & & & & \\
\hline & & & & \\
\hline Lunch & & & & \\
\hline & & & & \\
\hline & & & & \\
\hline Snack & & & & \\
\hline & & & & \\
\hline
\end{tabular}




\begin{tabular}{|l|l|l|l|l|}
\hline Dinner & & & & \\
\hline & & & & \\
\hline & & & & \\
\hline & & & & \\
\hline Evening & & & & \\
\hline & & & & \\
\hline & & & & \\
\hline
\end{tabular}




\section{5-Step Multiple-Pass Method}

$\begin{array}{ll}1 \text { Quick List } & \text { - Simple list of all foods eaten } \\ \text { - No order to list } & \text { - Specify a 24-hour period } \\ & \text { - One food/drink per line }\end{array}$

\section{4-Hour Food Recall Forgotten Foods}

There are some foods that people tend to forget they ate.

- Did you have any crackers, breads, rolls, or tortillas that you may have forgotten about?

- How about any hot or cold cereals?

- Cheese added as topping on vegetables or on a sandwich?

- Did you have any chips, candy, nuts, or seeds?

- Fruit eaten with meals or as a snack?

- What about coffee, tea, soft drinks, or juices?

- Any beer, wine, cocktails, brandies, or any other drinks made with liquor that you may have forgotten? 


\section{APPENDIX IV}

\section{Data Collection Sheet}

Squat limb:

Leg Extension Limb:

Exercise Order: (1)

Descriptors/DV's:

\begin{tabular}{|c|c|c|}
\hline Age & & \\
\hline Gender & & \\
\hline Training Status (Starting) & & \\
\hline Height & $\mathrm{cm}$ & in \\
\hline Weight & kg & lb \\
\hline BMI & & $\mathrm{Kg} / \mathrm{m}^{2}$ \\
\hline Body Fat \% & & $\%$ \\
\hline Thigh length (Left) & $\mathbf{c m}$ & in \\
\hline Thigh length (Right) & $\mathrm{cm}$ & in \\
\hline PRE Squat 1RM & kg & Ib \\
\hline PRE Leg Extension 1RM & $\mathbf{k g}$ & Ib \\
\hline POST Squat 1RM & kg & Ib \\
\hline POST Leg Extension 1RM & $\mathbf{k g}$ & Ib \\
\hline
\end{tabular}


APPENDIX V

Intervention and Strength Testing Data Collection Sheets

\begin{tabular}{|c|c|c|c|c|c|}
\hline Week & Training Goal & Sets & Reps & Load (\%1RM) & Rest (s) \\
\hline 1 & Hypertrophy & 3 & 12 & 50 & 90 \\
\hline 2 & Hypertrophy & 3 & 10 & 75 & 90 \\
\hline 3 & Hypertrophy & 3 & 8 & 80 & 90 \\
\hline 4 & Strength & 4 & 6 & 85 & 120 \\
\hline 5 & Strength & 4 & 6 & 85 & 120 \\
\hline 6 & Hypertrophy & 3 & 12 & 67 & 90 \\
\hline 7 & Hypertrophy & 3 & 10 & 75 & 90 \\
\hline 8 & Hypertrophy & 3 & 8 & 80 & 90 \\
\hline
\end{tabular}

1RM Testing Protocol (Date:

to

\begin{tabular}{|l|l|l|l|}
\hline Set & Estimated Intensity & Repetitions & Rest \\
\hline $\begin{array}{l}\text { Cycling } \\
(60 \mathrm{rpm})\end{array}$ & $0.5 \mathrm{kp}$ & 2.5 minutes & N/A \\
\hline
\end{tabular}




\begin{tabular}{|l|l|l|l|}
\hline Knee Hug & N/A & 8 & N/A \\
\hline Quad Pull & N/A & 8 & N/A \\
\hline Leg Swing & N/A & 8 & N/A \\
\hline Bodyweight & N/A & 8 & 1 \\
\hline 1 & $\begin{array}{l}50 \% \text { (Assume this is } \\
\text { the bar weight) }\end{array}$ & $5-6$ & 1 \\
\hline 2 & $\%-90 \%$ & $4-12$ & $2-3$ \\
\hline 3 & $65 \%-90 \%$ & $4-12$ & $2-3$ \\
\hline
\end{tabular}

Bike Height:

Squat limb: Squat bar depth: Heel

Placement: (In)

Leg Extension Limb: Tripod height:

Backrest holes visible in front (towards person): $(0-3)$

Backrest holes visible in back (away from person): $(0-2)$

Limb Order: (1) (D or ND) (2) (D or ND)

Limb Order: (1) (L or R) (2) ( $\mathrm{L}$ or $\mathrm{R})$

Exercise Order: (1) (2) 


\section{Squat}

Load: , Rep Number Hit:

1RM:

(From Chart)

\section{Leg Extension}

Load: , Rep Number Hit:

1RM:

(From Chart)

\section{Squat:}

First 1RM:

New 1RM*: (Date increase begins: )$^{* *}$

New 1RM*: (Date increase begins:

()$^{\star *}$

New 1RM*: (Date increase begins: )$^{\star \star}$

New 1RM*: (Date increase begins: )$^{\star \star}$

New 1RM*: (Date increase begins: )$^{* \star}$ 
New 1RM*: (Date increase begins:

${ }^{\star \star *}$

New 1RM*: (Date increase begins:

()$^{* *}$

New 1RM*: (Date increase begins: )$^{* *}$

New 1RM*: (Date increase begins:

()$^{\star \star}$

New 1RM*: (Date increase begins: )$^{\star \star}$

New $1 \mathrm{RM}^{*}$ : (Date increase begins:

$u^{* *}$

New 1RM*: (Date increase begins: )$^{\star \star}$

New 1RM*: (Date increase begins:

\section{Leg Extension:}

First 1RM:

New 1RM*: (Date increase begins: )$^{* *}$ 
New 1RM*: (Date increase begins: ${ }^{\star \star *}$

New 1RM*: (Date increase begins:

()$^{* *}$

New 1RM*: (Date increase begins: )$^{* *}$

New 1RM*: (Date increase begins:

()$^{\star \star}$

New 1RM*: (Date increase begins: )$^{\star \star}$

New 1RM*: (Date increase begins: $u^{* *}$

New 1RM*: (Date increase begins: )$^{\star \star}$

New 1RM*: (Date increase begins:

$\longrightarrow^{* *}$

New 1RM*: (Date increase begins: )$^{\star *}$

New 1RM*: (Date increase begins: )$^{* *}$

New 1RM*: (Date increase begins: )$^{* *}$ 
New $1 \mathrm{RM}^{*}$ : (Date increase begins:

)$^{* *}$

* $5 \%$ greater than previous $1 \mathrm{RM}$ (rounded up), only increase following 2 for 2 rule. Only update during training, NOT on 1 RM testing day

** This is the date training load calculations become based off of the new $1 \mathrm{RM}$ value as determined by the 2 for 2 rule.

WEEK 1 (Date: to

$1 \mathrm{RM}$ for week:

\begin{tabular}{|c|c|c|c|c|c|}
\hline Week & Training Goal & Sets & Reps & Load (\%1RM) & Rest (s) \\
\hline 1 & Hypertrophy & 3 & 12 & 50 & 90 \\
\hline
\end{tabular}

Squat Load: $(1 \mathrm{RM} \times 0.50)$

Leg Extension Load: $(1 \mathrm{RM} \times 0.50)$

SQUAT

\begin{tabular}{|l|l|l|l|}
\hline Da & Set 1 Reps & Set 2 Reps & Set 3 Reps \\
$y$ & & & \\
\hline
\end{tabular}




\begin{tabular}{|c|l|l|l|}
\hline 1 & $X X X X X X X X X X X X X X$ & $X X X X X X X X X X X X X X$ & $X X X X X X X X X X X$ \\
& $x$ & $X$ & $X X X X$ \\
\hline 2 & & & \\
\hline 3 & & & \\
\hline
\end{tabular}

Squat Notes:

\section{Leg Extension}

\begin{tabular}{|c|l|l|l|}
\hline Day & Set 1 Reps & Set 2 Reps & Set 3 Reps \\
\hline 1 & XXXXXXXXXXXXXX & XXXXXXXXXXXXXX & XXXXXXXXXXXX \\
& & & $X X$ \\
\hline 2 & & & \\
\hline 3 & & & \\
\hline
\end{tabular}


Leg Extension Notes:

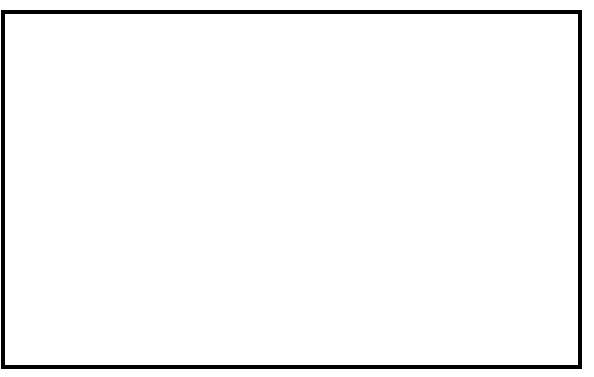

WEEK 2 (Date:

to

$1 \mathrm{RM}$ for week:

\begin{tabular}{|c|c|c|c|c|c|}
\hline Week & Training Goal & Sets & Reps & Load (\%1RM) & Rest (s) \\
\hline 2 & Hypertrophy & 3 & 10 & 75 & 90 \\
\hline
\end{tabular}

Squat Load: $(1 \mathrm{RM} \times 0.75)$

Leg Extension Load: $(1 \mathrm{RM} \times 0.75)$

SQUAT

\begin{tabular}{|c|l|l|l|}
\hline Day & Set 1 Reps & Set 2 Reps & Set 3 Reps \\
\hline 1 & & & \\
\hline
\end{tabular}




\begin{tabular}{|l|l|l|l|}
\hline 2 & & & \\
\hline 3 & & & \\
\hline
\end{tabular}

Squat Notes:

\section{Leg Extension}

\begin{tabular}{|c|l|l|l|}
\hline Day & Set 1 Reps & Set 2 Reps & Set 3 Reps \\
\hline 1 & & & \\
\hline 2 & & & \\
\hline 3 & & & \\
\hline
\end{tabular}

Leg Extension Notes: 
WEEK 3 (Date: to $1 \mathrm{RM}$ for week:

\begin{tabular}{|c|c|c|c|c|c|}
\hline Week & Training Goal & Sets & Reps & Load (\%1RM) & Rest (s) \\
\hline 3 & Hypertrophy & 3 & 8 & 80 & 90 \\
\hline
\end{tabular}

Squat Load: $(1 \mathrm{RM} \times 0.80)$

Leg Extension Load: $(1 \mathrm{RM} \times 0.80)$

\section{SQUAT}

\begin{tabular}{|c|l|l|l|}
\hline Day & Set 1 Reps & Set 2 Reps & Set 3 Reps \\
\hline 1 & & & \\
\hline 2 & & & \\
\hline 3 & & & \\
\hline
\end{tabular}


Squat Notes:

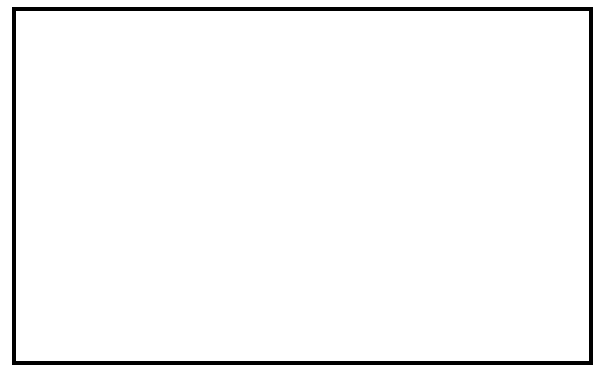

\section{Leg Extension}

\begin{tabular}{|c|l|l|l|}
\hline Day & Set 1 Reps & Set 2 Reps & Set 3 Reps \\
\hline 1 & & & \\
\hline 2 & & & \\
\hline 3 & & & \\
\hline
\end{tabular}

Leg Extension Notes: 
WEEK 4 (Date: to $1 \mathrm{RM}$ for week:

\begin{tabular}{|c|c|c|c|c|c|}
\hline Week & Training Goal & Sets & Reps & Load (\%1RM) & Rest (s) \\
\hline 4 & Strength & 4 & 6 & 85 & 120 \\
\hline
\end{tabular}

Squat Load: $(1 \mathrm{RM} \times 0.85)$

Leg Extension Load: $(1 \mathrm{RM} \times 0.85)$

\section{SQUAT}

\begin{tabular}{|c|l|l|l|l|}
\hline Day & Set 1 Reps & Set 2 Reps & Set 3 Reps & Set 4 Reps \\
\hline 1 & & & & \\
\hline 2 & & & & \\
\hline 3 & & & & \\
\hline
\end{tabular}

Squat Notes: 


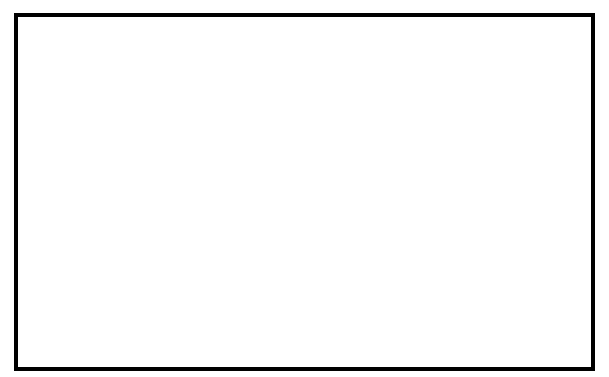

Leg Extension

\begin{tabular}{|c|l|l|l|l|}
\hline Day & Set 1 Reps & Set 2 Reps & Set 3 Reps & Set 4 Reps \\
\hline 1 & & & & \\
\hline 2 & & & & \\
\hline 3 & & & & \\
\hline
\end{tabular}

Leg Extension Notes:

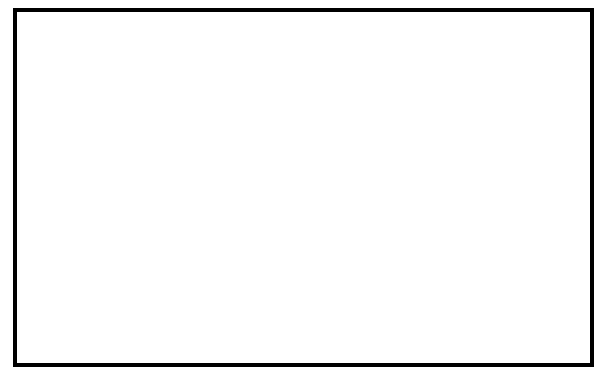

WEEK 5 (Date:

to

) $1 \mathrm{RM}$ for

week: 


\begin{tabular}{|c|c|c|c|c|c|}
\hline Week & Training Goal & Sets & Reps & Load (\%1RM) & Rest (s) \\
\hline 5 & Strength & 4 & 6 & 85 & 120 \\
\hline
\end{tabular}

Squat Load:

$(1 \mathrm{RM} \times 0.85)$

Leg Extension Load:

$(1 \mathrm{RM} \times 0.85)$

\section{SQUAT}

\begin{tabular}{|c|l|l|l|l|}
\hline Day & Set 1 Reps & Set 2 Reps & Set 3 Reps & Set 4 Reps \\
\hline 1 & & & & \\
\hline 2 & & & & \\
\hline 3 & & & & \\
\hline
\end{tabular}

Squat Notes:

\section{Leg Extension}




\begin{tabular}{|c|l|l|l|l|}
\hline Day & Set 1 Reps & Set 2 Reps & Set 3 Reps & Set 4 Reps \\
\hline 1 & & & & \\
\hline 2 & & & & \\
\hline 3 & & & & \\
\hline
\end{tabular}

Leg Extension Notes:

WEEK 6 (Date: to

$1 \mathrm{RM}$ for week:

\begin{tabular}{|c|c|c|c|c|c|}
\hline Week & Training Goal & Sets & Reps & Load (\%1RM) & Rest (s) \\
\hline 6 & Hypertrophy & 3 & 12 & 67 & 90 \\
\hline
\end{tabular}

Squat Load: $(1 \mathrm{RM} \times 0.67)$

Leg Extension Load: $(1 \mathrm{RM} \times 0.67)$ 
SQUAT

\begin{tabular}{|c|l|l|l|}
\hline Day & Set 1 Reps & Set 2 Reps & Set 3 Reps \\
\hline 1 & & & \\
\hline 2 & & & \\
\hline 3 & & & \\
\hline
\end{tabular}

Squat Notes:

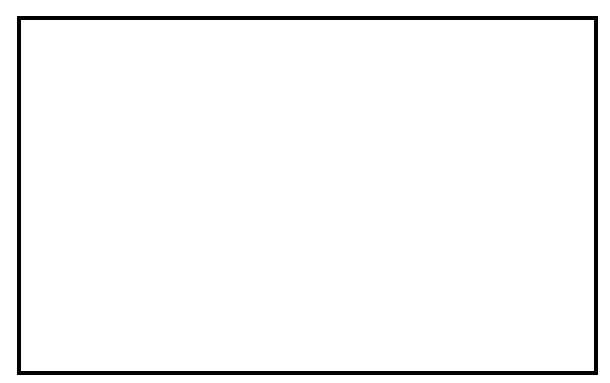

Leg Extension

\begin{tabular}{|c|l|l|l|}
\hline Day & Set 1 Reps & Set 2 Reps & Set 3 Reps \\
\hline 1 & & & \\
\hline 2 & & & \\
\hline 3 & & & \\
\hline
\end{tabular}


Leg Extension Notes:

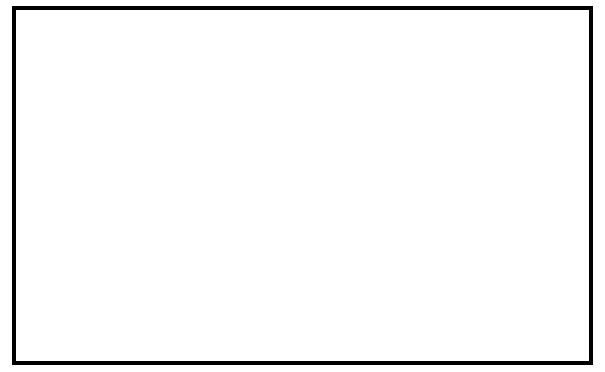

WEEK 7 (Date:

to

$1 \mathrm{RM}$ for

week:

\begin{tabular}{|c|c|c|c|c|c|}
\hline Week & Training Goal & Sets & Reps & Load (\%1RM) & Rest (s) \\
\hline 7 & Hypertrophy & 3 & 10 & 75 & 90 \\
\hline
\end{tabular}

Squat Load:

$(1 \mathrm{RM} \times 0.75)$

Leg Extension Load:

$(1 \mathrm{RM} \times 0.75)$

SQUAT

\begin{tabular}{|c|l|l|l|}
\hline Day & Set 1 Reps & Set 2 Reps & Set 3 Reps \\
\hline 1 & & & \\
\hline
\end{tabular}




\begin{tabular}{|l|l|l|l|}
\hline 2 & & & \\
\hline 3 & & & \\
\hline
\end{tabular}

Squat Notes:

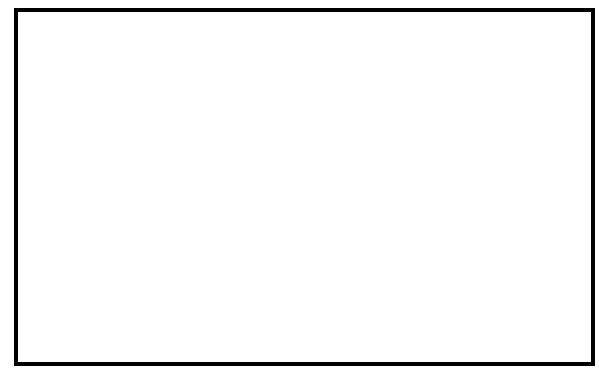

\section{Leg Extension}

\begin{tabular}{|c|l|l|l|}
\hline Day & Set 1 Reps & Set 2 Reps & Set 3 Reps \\
\hline 1 & & & \\
\hline 2 & & & \\
\hline 3 & & & \\
\hline
\end{tabular}

Leg Extension Notes:

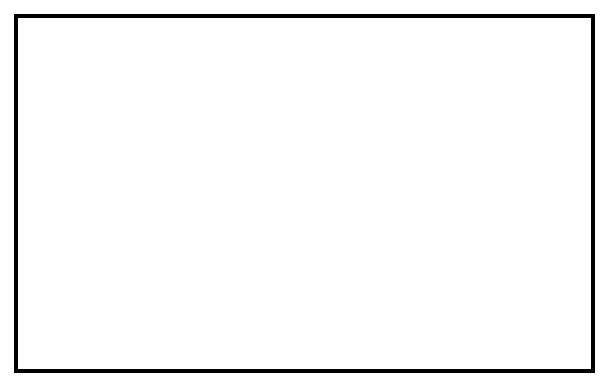


WEEK 8 (Date:

to

1RM for

week:

\begin{tabular}{|c|c|c|c|c|c|}
\hline Week & Training Goal & Sets & Reps & Load (\%1RM) & Rest (s) \\
\hline 8 & Hypertrophy & 3 & 8 & 80 & 90 \\
\hline
\end{tabular}

Squat Load:

$(1 \mathrm{RM} \times 0.80)$

Leg Extension Load:

$(1 \mathrm{RM} \times 0.80)$

SQUAT

\begin{tabular}{|c|l|l|l|}
\hline Day & Set 1 Reps & Set 2 Reps & Set 3 Reps \\
\hline 1 & & & \\
\hline 2 & & & \\
\hline 3 & & & \\
\hline
\end{tabular}

Squat Notes: 


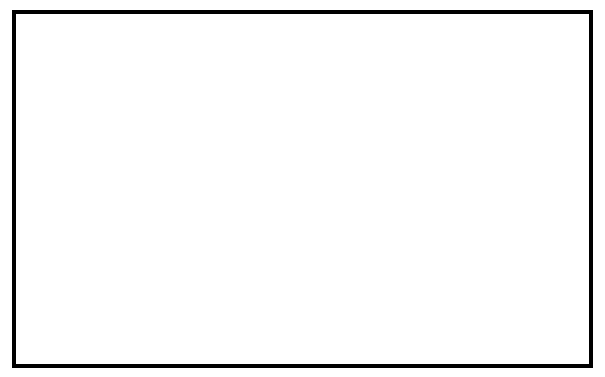

Leg Extension

\begin{tabular}{|c|l|l|l|}
\hline Day & Set 1 Reps & Set 2 Reps & Set 3 Reps \\
\hline 1 & & & \\
\hline 2 & & & \\
\hline 3 & & & \\
\hline
\end{tabular}

Leg Extension Notes:

Post Testing 1RM Testing Protocol (Date: to

\begin{tabular}{|l|l|l|l|}
\hline Set & Estimated Intensity & Repetitions & Rest \\
\hline
\end{tabular}




\begin{tabular}{|l|l|l|l|}
\hline Cycling (60rpm) & $0.5 \mathrm{kp}$ & 2.5 minutes & N/A \\
\hline Knee Hug & N/A & 8 & N/A \\
\hline Quad Pull & N/A & 8 & N/A \\
\hline Leg Swing & N/A & 8 & N/A \\
\hline Sodyweight & N/A & 8 & 1 \\
\hline 1 & $\begin{array}{l}50 \% \text { (Assume this is } \\
\text { the bar weight) }\end{array}$ & $5-6$ & 1 \\
\hline 2 & $65 \%-90 \%$ & $4-12$ & $2-3$ \\
\hline 3 & $65 \%-90 \%$ & $4-12$ & $2-3$ \\
\hline
\end{tabular}

Only count sets where failure occurs between 8-12 reps:

\section{Squat}

Load: Rep Number Hit: 1RM:

(From Chart)

\section{Leg Extension}

Load: , Rep Number Hit: 1RM:

(From Chart) 


\section{APPENDIX VI}

\section{Borg CR-10 Scale of Perceived Exertion}

While exercising we want you to rate your perception of exertion, i.e., how heavy and strenuous the exercise feels to you. The perception of exertion depends mainly on the strain and fatigue in you muscles and on your feeling of breathlessness or aches in the chest. Look at this rating scale; we want you to use this scale from 1 to 10 , where 1 means "no exertion at all" and 10 means "maximal or very, very strong exertion." For most people this is the most strenuous resistance exercise they have ever experienced. Try to appraise your feeling of exertion as honestly as possible, without thinking about what the actual physical load is. Don't underestimate it, but don't overestimate it either. It's your own feeling of effort and exertion that's important, not how it compares to other people's. What other people think is not important either. In addition, this scale has no anchor. That is, if after giving a "10" on a previous rating, you decide that the current exercise is more strenuous, you may give a higher number (i.e. "11"0. Look at the scale and the expressions and then give a number.

Any questions? 


\begin{tabular}{|lll|}
\hline & \multicolumn{1}{c|}{ Borg CR-10 Scale of Perceived Exertion } \\
0 & Nothing at all & \\
0.3 & & \\
0.5 & Extremely weak & Just noticeable \\
0.7 & \\
1 & Very weak & \\
1.5 & \\
2 & Weak & \\
2.5 & \\
3 & Moderate & \\
4 & & \\
5 & Strong & \\
6 & & \\
7 & Very strong & \\
8 & & \\
9 & & \\
10 & Extremely strong & \\
11 & & \\
\hline & & \\
\hline
\end{tabular}




\section{APPENDIX VII \\ Email Recruitment Script}

Dear [name],

A new and exciting research study is being conducted by the Department of Kinesiology at the University of Rhode Island! This study is currently seeking willing participants of any gender between the ages of 18-35 who do not regularly resistance train their lower body to engage in lower body resistance training. The study will last approximately 10.5 hours over 9 weeks and compensation of $\$ 250$ will be awarded to any participants that attend all training and testing sessions.

Eligible participants will not have regularly resistance trained their lower body for 2 or more days per week over the most recent 6 months.

If you are interested in learning more and potentially participating in this study, please reach out to the research team at URIQuadricepsStudy@gmail.com.

Sincerely,

The URI Quadriceps Study research team

Principle Investigator Jacob Earp PhD, CSCS

Jacob earp@uri.edu

401-874-7845 


\section{APPENDIX VIII}

\section{Recruitment Flyer}

\section{KINESIOLOGY RESEARCH OPPORTUNITY!!}

ARE YOU BETWEEN THE AGES OF 18 AND 35?

We are looking for subjects to participste in our study on quadriceps growth using various lower body evercises

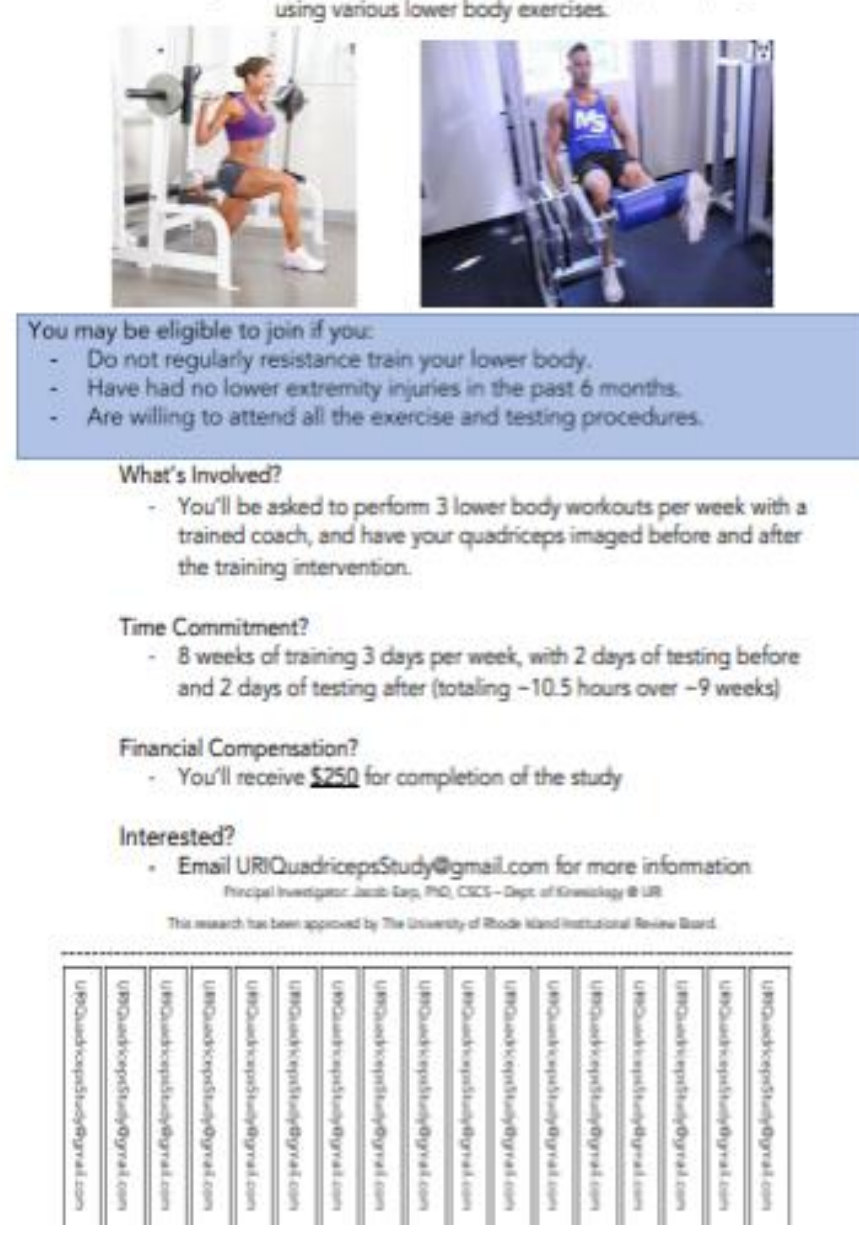




\section{BIBLIOGRAPHY}

1. Escamilla RF, Fleisig GS, Zheng N, Barrentine SW, Wilk KE, Andrews JR. Biomechanics of the knee during closed kinetic chain and open kinetic chain exercises. Med Sci Sports Exerc. 1998;30(4):556-69.

2. Hakkinen K, Newton RU, Gordon SE, et al. Changes in Muscle Morphology, Electromyographic Activity, and Force Production Characteristics During Progressive Strength Training in Young and Older Men. 1998. 415-423 p. [cited 2019 Mar 2 ] Available from: https://academic.oup.com/biomedgerontology/articleabstract/53A/6/B415/573497.

3. Blazevich AJ, Gill ND, Zhou S. Intra- and intermuscular variation in human quadriceps femoris architecture assessed in vivo. $J$ Anat. 2006;209(3):289-310.

4. Waligora AC, Johanson NA, Hirsch BE. Clinical Anatomy of the Quadriceps Femoris and Extensor Apparatus of the Knee. Clin Orthop Relat Res. 2009;467(12):3297-306.

5. Maughan RJ, Watson JS, Weir J. Strength and cross-sectional area of human skeletal muscle. J Physiol. 1983;338(1):37-49.

6. Kumagai K, Abe T, Brechue WF, Ryushi T, Takano S, Mizuno M. Sprint performance is related to muscle fascicle length in male 100-m sprinters. J Appl Physiol. 2000;88(3):811-6.

7. Mujika I, Santisteban J, Impellizzeri FM, Castagna C. Fitness determinants of success in men's and women's football. J Sports Sci. 
$2009 ; 27(2): 107-14$.

8. Hughes PE, Hsu JC, Matava MJ. Hip Anatomy and Biomechanics in the Athlete [Internet]. 2002 [cited 2019 Jun 6 ]; available from:

https://pdfs.semanticscholar.org/117a/4a6f0fab932103ccdd7dc6c7aeec 37f8fc00.pdf. doi:10.1097/01.JSA.0000017305.66476.DA.

9. Fletchern JR, Maclntosh BR. Running economy from a muscle energetics perspective. Front Physiol. 2017;8(JUN):433.

10. Byrne DP, Mulhall KJ, Baker JF. Anatomy \&amp; Biomechanics of the Hip. 2010. 51-57 p. [cited 2019 Jun 6 ] Available from:

https://benthamopen.com/contents/pdf/TOSMJ/TOSMJ-4-51.pdf.

11. Earp JE, Newton RU, Cormie P, Blazevich AJ. Inhomogeneous Quadriceps Femoris Hypertrophy in Response to Strength and Power Training. Med Sci Sports Exerc. 2015;47(11):2389-97.

12. Ema R, Wakahara T, Miyamoto N, Kanehisa H, Kawakami Y. Inhomogeneous architectural changes of the quadriceps femoris induced by resistance training. Eur J Appl Physiol. 2013;113(11):2691703.

13. Miyamoto N, Wakahara T, Kawakami Y. Task-Dependent Inhomogeneous Muscle Activities within the Bi-Articular Human Rectus Femoris Muscle. PLoS One. 2012;7(3):e34269.

14. Wakahara T, Ema R, Miyamoto N, Kawakami Y. Inter- and intramuscular differences in training-induced hypertrophy of the quadriceps femoris: association with muscle activation during the first 
training session. Clin Physiol Funct Imaging. 2017;37(4):405-12.

15. Browning RC, Modica JR, Kram R, Goswami A, Browning R. The Effects of Adding Mass to the Legs on the Energetics and Biomechanics of Walking. Biomech Walking Med Sci Sport Exerc. 2007;39(3):515-25.

16. Lossifidou AN, Baltzopoulos V. Inertial Effects on During Isokinetic Moment Development Concentric nee-Extension Testing. 2000. 317327 p. [cited 2019 Jun 6 ] Available from: www.jospt.org.

17. WAKAHARA T, FUKUTANI A, KAWAKAMI Y, YANAI T. Nonuniform Muscle Hypertrophy. Med Sci Sport Exerc. 2013;45(11):2158-65.

18. STENSDOTTER A-K, HODGES PW, MELLOR R, SUNDELIN G, H??GER-ROSS C. Quadriceps Activation in Closed and in Open Kinetic Chain Exercise. Med Sci Sport Exerc. 2003;35(12):2043-7.

19. Schoenfeld BJ. Squatting Kinematics and Kinetics and Their Application to Exercise Performance. J Strength Cond Res. 2010;24(12):3497-506.

20. Hahn D. Lower Extremity Extension Force and Electromyography Properties as a Function of Knee Angle and Their Relation to Joint Torques: Implications for Strength Diagnostics. J Strength Cond Res. $2011 ; 25(6): 1622-31$.

21. Daviin CD, Holcomb WR, Guadagnoli MA. The Effect of Hip Position and Electromyographic Biofeedback Training on the Vastus Medialis Oblique: Vastus Lateralis Ratio. 1999. 342-349 p. [cited 2019 Jul 8 ] Available from: www.nata.org/jat.

22. Abe $T$, Kumagai $K$, Brechue WF. Fascicle length of leg muscles is 
greater in sprinters than distance runners. Med Sci Sports Exerc. 2000;32(6):1125-9.

23. Abe $\mathrm{T}$, Kumagai $\mathrm{K}$, Brechue $\mathrm{W}$. Fascicle length of leg muscles is greater in sprinters than distance runners. Med Sci Sport Exerc. 2000;1125-9.

24. Hudson AL, Gandevia SC, Butler JE. A Principle of Neuromechanical Matching for Motor Unit Recruitment in Human Movement. Exerc Sport Sci Rev. 2019;1.

25. Giles LS, Webster KE, McClelland JA, Cook J. Does Quadriceps Atrophy Exist in Individuals With Patellofemoral Pain? A Systematic Literature Review With Meta-analysis [Internet]. J Orthop Sport Phys Ther. 2013; doi:10.2519/jospt.2013.4833.

26. Witvrouw E, Pt † , Lysens R, Bellemans J, Peers K, Vanderstraeten G. Open Versus Closed Kinetic Chain Exercises for Patellofemoral Pain A Prospective, Randomized Study. 2000. [cited 2019 Mar 2 ] Available from:

https://journals.sagepub.com/doi/pdf/10.1177/03635465000280051201? casa_token=mwuV9myQ-wEAAAAA:ZMuAgLbuHpKMzSgy_QFE_hU8r4WBkf4sVDofAS7TJku6ObBFCEc36G_RYx 68vPQtXilZ5xLes0.

27. Mitchell J, De D, Original Article K. A comparison of the proximal and distal parts of the vastus medialis muscle. 1997. 277-281 p. [cited 2019 Mar 2 ] Available from: https://ac.els-cdn.com/S0004951414604165/1s2.0-S0004951414604165-main.pdf?_tid=f0044ee8-f14d-4654-912b- 
bff $1 \mathrm{fc} 0632 \mathrm{e} 2 \&$ acdnat $=1551552529 \_879425161 \mathrm{a} 08 \mathrm{ba3840e5d496161a}$ aa0c.

28. Wilk KE, Escamilla RF, Fleisig GS, Barrentine SW, Andrews JR, Boyd ML. A Comparison of Tibiofemoral Joint Forces and Electromyographic Activit During Open and Closed Kinetic Chain Exercises. Am J Sports Med. 1996;24(4):518-27.

29. Azizi E, Brainerd EL, Roberts TJ. Variable gearing in pennate muscles. 2008. [cited 2019 Jul 8 ] Available from: www.pnas.orgcgidoi10.1073pnas.0709212105.

30. Peters JSJ, Tyson NL. Proximal exercises are effective in treating patellofemoral pain syndrome: a systematic review. Int J Sports Phys Ther. 2013;8(5):689-700.

31. Half G, Triplett T. Essentials of Strength Training and Conditioning 4th Edition - Google Books. 4th ed. Champaign: Human Kinetics; 2016. 735 p. [cited 2019 Jun 6 ] Available from:

https://books.google.com/books?hl=en\&lr=\&id=bfuXCgAAQBAJ\&oi=fnd \&pg=PR $1 \& d q=$ essentials+of + strength + and + conditioning +4 th\&ots=2ers 6 vzXWz\&sig=hOUxRkOejolzdb3oy12iLIRA4tg\#v=onepage\&q=essentials of strength and conditioning 4 th \&f=false.

32. Zhang G, Kacer M, Lane AH, Chen JJ, Wilson TA. Effect of Inspiration/Expiration on Height Measurement PILI 'Ohana Project: Partnership to Overcome Obesity Disparities in Hawai'i View project Effect of Inspiration/Expiration on Height Measurement. Artic J Pediatr 
Endocrinol Metab JPEM. 2008;21:763-9.

33. Jackson AS, Mahar MT. Reliability and validity of bioelectrical impedance in determining body composition [Internet]. Artic J Appl Physiol. 1988 [cited 2019 Jun 6 ]; available from: https://www.researchgate.net/publication/19784744. doi:10.1152/jappl.1988.64.2.529.

34. Oppliger RA, Magnes SA, Popowski LA, Gisolfi C V. Accuracy of Urine Specific Gravity and Osmolality as Indicators of Hydration Status. Int $J$ Sport Nutr Exerc Metab. 2005;15(3):236-51.

35. Klatt TD, Knight KL, Hogan ZL, Leon EE, Meyer KA. Changes in Urine Specific Gravity and Bioelectrical Impedance Analysis Between NormalHydrated and Euhydrated States [Internet]. 2009 [cited 2019 Jun 6 ]; available from: https://escholarshare.drake.edu/handle/2092/947.

36. Moloney ) -S C, Lemmey AB. Effects of hydration state and rehydration method on bioelectrical impedance analysis A B Lemmey (BEd (PE), Hons (Exercise Physiol), MA, PhD) R G Eston (BEd (Hons) CORRESPONDENCE. [date unknown]. [cited 2019 Jun 6 ] Available from:

https://journals.co.za/docserver/fulltext/m_sajsm/7/1/1319.pdf?expires=1 $559831511 \& i d=i d \& a c c n a m e=$ guest $\&$ checksum $=76$ EA0157D0A10687D D5258E731DD844F.

37. Tomaras EK, Macintosh B. Less is more: Standard warm-up causes fatigue and less warm-up permits greater cycling power output Energy 
cost of running at high intensity View project [Internet]. Artic J Appl Physiol. 2011 [cited 2019 Jun 6 ]; available from:

https://www.researchgate.net/publication/51106401.

doi:10.1152/japplphysiol.00253.2011.

38. Barroso R, Silva-Batista C, Tricoli V, Roschel H, Ugrinowitsch C. The Effects of Different Intensities and Durations of the General Warm-up on Leg Press 1RM. J Strength Cond Res. 2013;27(4):1009-13.

39. Signorile JF, Kacsik D, Perry A, et al. The Effect of Knee and Foot Position on the Electromyographical Activity of the Superficial Quadriceps. 1995. [cited 2020 Mar 8 ] Available from: www.jospt.org.

40. Verdijk LB, van Loon L, Meijer K, Savelberg HHCM. One-repetition maximum strength test represents a valid means to assess leg strength in vivo in humans. J Sports Sci. 2009;27(1):59-68.

41. Kanyakham MK. The measurement of transverse relaxation time (T 2 ) using histogram from ROI setting in muscle activity study at 1.5 Tesla $M R I$. [date unknown].

42. Norton J, Donaldson N, Dekker L. 3D whole body scanning to determine mass properties of legs. J Biomech. 2002;35(1):81-6.

43. Nordez A, Jolivet E, Südhoff I, Bonneau D, de Guise JA, Skalli W. Comparison of methods to assess quadriceps muscle volume using magnetic resonance imaging. J Magn Reson Imaging. 2009;30(5):111623.

44. Bird SP, Marino F. Designing Resistance Training Programmes to 
Enhance Muscular Fitness Article in Sports Medicine · February 2005 [Internet]. 2016 [cited 2020 Apr 29 ]; available from:

https://www.researchgate.net/publication/7583080.

doi:10.2165/00007256-200535100-00002.

45. Dugan SA, Bhat KP. Biomechanics and Analysis of Running Gait [Internet]. [date unknown]; doi:10.1016/j.pmr.2005.02.007.

46. Yoshioka S, Nagano A, Hay DC, Fukashiro S. Biomechanical analysis of the relation between movement time and joint moment development during a sit-to-stand task. Biomed Eng Online. 2009;8(1):27.

47. Schoenfeld BJ. The Mechanisms of Muscle Hypertrophy and Their Application to Resistance Training. J Strength Cond Res. 2010;24(10):2857-72.

48. Paoli A, Gentil P, Moro T, Marcolin G, Bianco A. Resistance Training with Single vs. Multi-joint Exercises at Equal Total Load Volume: Effects on Body Composition, Cardiorespiratory Fitness, and Muscle Strength. Front Physiol. 2017;8(DEC):1105.

49. Wakahara T, Miyamoto N, Sugisaki N, et al. Association between regional differences in muscle activation in one session of resistance exercise and in muscle hypertrophy after resistance training. Eur J Appl Physiol. 2012;112(4):1569-76.

50. Prietto CA, Caiozzo VJ. The in vivo force-velocity relationship of the knee flexors and extensors. [date unknown].

51. Dellanini L, Hawkins D, Martin RB, Stover S. An investigation of the 
interactions between lower-limb bone morphology, limb inertial properties and limb dynamics. J Biomech. 2003;36(7):913-9.

52. Kukolj M, Ropret R, Ugarkovic D, Jaric S. Anthropometric, strength, and power predictors of sprinting performance. J Sports Med Phys Fitness. 1999;39(2):120-2.

53. D.J. C, D.J. W, N.J. O, et al. Strength and power predictors of sprinting performance in professional rugby players. J Sports Med Phys Fitness. 2013;53(2):105-11.

54. Williams MA. THE EFFECT OF MENSTRUAL CYCLE PHASE ON MAXIMAL STRENGTH AND PERCEPTION OF EXERTION. 1993.

55. Mihm M, Gangooly S, Muttukrishna S. The normal menstrual cycle in women. Anim Reprod Sci. 2011;124(3-4):229-36.

56. Bishop P, Green JM. Cross-Sectional Area and Muscular Strength [Internet]. 2014 [cited 2020 Mar 8 ]; available from:

https://www.researchgate.net/publication/23489962.

doi:10.2165/00007256-200838120-00003.

57. Nicola TL, Jewison DJ. The Anatomy and Biomechanics of Running [Internet]. 2012; doi:10.1016/j.csm.2011.10.001.

58. Johnson MD, Buckley JG. Muscle power patterns in the midacceleration phase of sprinting [Internet]. 2010 [cited 2020 Mar 8 ]; available from:

https://www.tandfonline.com/action/journallnformation?journalCode=rjsp 20. doi:10.1080/026404101750158330. 
59. Myers MJ, Steudel K. Effect of limb mass and its distribution on the energetic cost of running. J Exp Biol. 1985;VOL. 116:363-73.

60. Mero AA, Gregor R. Biomechanics of Sprint Running Exercise and Inflammation with Special Reference to Resistance Training View project Sensorimotor control of locomotion after peripheral nerve injury View project [Internet]. 1992 [cited 2020 Mar 8 ]; available from: https://www.researchgate.net/publication/21540989. doi:10.2165/00007256-199213060-00002.

61. Hamner SR, Seth A, Delp SL. Muscle contributions to propulsion and support during running. J Biomech. 2010;43(14):2709-16.

62. Relationships between Ground Reaction Force Impulse and Kinematics of Sprint-Running Acceleration [Internet]2005 [cited 2020 Mar 8 ]; available from: https://www.researchgate.net/publication/7628671. doi:10.1123/jab.21.1.31.

63. Martin SB, Morrow JR, Jackson AW, Dunn AL. Variables related to meeting the CDC/ACSM physical activity guidelines. 2000. 2087-2092 p. [cited 2020 Apr 29 ] Available from: http://www.acsm-msse.org.

64. Negrete R, Health $\mathrm{O}$. The Relationship between Isokinetic Open and Closed Chain Lower Extremity Strength and Functional Performance [Internet]. 2000 [cited 2020 Apr 29 ]; available from: https://www.researchgate.net/publication/286829358. doi:10.1123/jsr.9.1.46.

65. Thorn\& R, Sweden G. Weight Training of the Thigh Muscles Using 
Closed Vs. Open Kinetic Chain Exercises: A Comparison of Performance Enhancement. 1998. [cited 2020 Apr 29 ] Available from: www.jospt.org.

66. Burt D, Lamb K, Nicholas C. Effects of repeated bouts of squatting exercise on sub-maximal endurance running performance [Internet]. [date unknown]; doi:10.1007/s00421-012-2437-2.

67. Amis AA, Firer P, Mountney J, Senavongse W, Thomas NP. Anatomy and biomechanics of the medial patellofemoral ligament. Knee. 2003;10(3):215-20.

68. Cavanagh PR, Gregor RJ. Knee joint torque during the swing phase of normal treadmill walking. J Biomech. 1975;8(5):337-44.

69. Phillips SM, Hartman JW, Wilkinson SB. Dietary Protein to Support Anabolism with Resistance Exercise in Young Men. J Am Coll Nutr. 2005;24(2):134S-139S.

70. Van Loon LJC, Gibala MJ. Dietary Protein to Support Muscle Hypertrophy. Nestec Ltd., Vevey/S. Karger AG; 2011. 79-95 p. 